\title{
Turán problems for integer-weighted Graphs
}

\author{
Zoltán Füredi \\ DEPARTMENT OF MATHEMATICS, UNIVERSITY OF ILLINOIS \\ URBANA, ILLINOIS 61801 \\ RÉNYI INSTITUTE OF MATHEMATICS OF THE HUNGARIAN \\ $A C A D E M Y$, \\ 1364 BUDAPEST, PF. 127, HUNGARY \\ Email: z-furedi@math.uiuc.edu, furedi@renyi.hu

André Kündgen
DEPARTMENT OF MATHEMATICS, UNIVERSITY OF ILLINOIS
URBANA, IL 61801
CURRENT ADDRESS: DEPARTMENT OF MATHEMATICS,
CALIFORNIA STATE UNIVERSITY, SAN MARCOS, CA 92096
Email: akundgen@csusm.edu

Keywords: Multigraphs, weighted graphs, Turán's Theorem, density, integer lattice AMS Subject Classification: 05D99, 05C35

\begin{abstract}
A multigraph is $(k, r)$-dense if every $k$-set spans at most $r$ edges. What is the maximum number of edges $\operatorname{ex}_{\mathbb{N}}(n, k, r)$ in a $(k, r)$-dense multigraph on $n$ vertices? We determine the maximum possible weight of such graphs for almost all $k$ and $r$ (e.g., for all $r>k^{3}$ ) by determining a constant $m=m(k, r)$ and showing that $\operatorname{ex}_{\mathbb{N}}(n, k, r)=m\left(\begin{array}{l}n \\ 2\end{array}\right)+O(n)$, thus giving a generalization of Turán's theorem. We find exact answers in many cases, even when negative integer weights are also allowed. In fact, our main result is to determine the maximum weight of $(k, r)$-dense $n$-vertex multigraphs with arbitrary integer weights with an $O(n)$ error term. (c) 2/11/1999 rev 2/27/01, 8/25/01 John Wiley \& Sons, Inc.
\end{abstract}

Journal of Graph Theory Vol. SUBMITTED, 1-31 (2/11/1999 rev 2/27/01, 8/25/01)

(c) 2/11/1999 rev 2/27/01, 8/25/01 John Wiley \& Sons, Inc.

CCC ??? 


\section{JOURNAL OF GRAPH THEORY}

\section{INTRODUCTION}

Much work has been done in the last forty years on the following problem introduced by Erdös in 1963: what is the largest number $\operatorname{ex}(n, k, r)$ of edges in a graph on $n$ vertices such that every set of $k$ vertices spans at most $r$ edges? In the case when $r=\left(\begin{array}{c}k \\ 2\end{array}\right)-1$, for example, Turán's Theorem [43,44] completely answers the question. Many papers, such as $[16,22,23,27,28]$, have led to a better understanding of Erdös' problem, with much work being devoted to the asymptotic behaviour of ex $(n, k, r)$ when $n$ grows. For example, $[27,28]$ contains a discussion of the cases in which the growth is linear or quadratic in $n$.

Recently, an integer-weighted version of the problem has been investigated. Here integer weights are assigned to the edges and we ask for the largest total weight $\operatorname{ex}_{\mathbb{Z}}(n, k, r)$ that a graph on $n$ vertices can have such that every set of $k$ vertices spans a subgraph with total weight at most $r$. This problem, when negative weights are not allowed, can be viewed as a multigraph version of the original Erdös problem.

Clearly, $\operatorname{ex}_{\mathbb{Z}}(n, k, r) \geq \operatorname{ex}(n, k, r)$. Recently, Bondy and Tuza [5] proved, among other things, that in the Turán case $r=\left(\begin{array}{l}k \\ 2\end{array}\right)-1$ equality must hold. Thus allowing integer weights does not result in any gain. However, unlike in the graph case where there is a unique extremal configuration, they found that in the integer-weighted case there can be several non-isomorphic ways of achieving the extremum. Additional cases are solved in the thesis of Kuchenbrod [33] and the exact value of $\operatorname{ex}_{\mathbb{Z}}(n, k, r)$ is determined for $k \leq 7$.

In the present paper, we asymptotically determine $\operatorname{ex}_{\mathbb{Z}}(n, k, r)$ for every choice of $k$ and $r$. We offer a conjecture for the exact value which we can prove in "slightly more than $50 \%$ of the cases".

More precisely, we provide upper and lower bounds for $e x_{\mathbf{Z}}(n, k, r)$ that differ by at most an $\mathrm{O}(n)$-term, so our estimates (usually of order $\Theta\left(n^{2}\right)$ ) are asymptotically very tight. Frequently the constructions we give do not require negative weights, so that in many cases we simultaneously solve the multigraph version of Erdös' original problems as well.

This paper is organized as follows. In Section 2. we give a brief overview of our results and we mention an application to the Turán theory of hypergraphs. Section 3. contains a brief history of Erdős' problem for graphs, and in Section 4. we generalize the problem to weighted graphs. In Section 5. we state our main results, such as the limits in the case of arbitrary integer weights. In Section 6. a recursive upper bound is given. In Section 7. a construction is given with special attention to the linear error term (which in some cases is not exactly known). In Section 8. we provide exact answers for "slightly more than $50 \%$ of the cases". In Section 9 . we continue our investigation by proving a lower bound which we conjecture to be exact. In Section 10. we solve our density question for sufficiently large $n$ whenever the limit is 0 . In Section 11 . we apply our results for integer weighted graphs to the multigraph case. We conclude the paper in Section 12. by proposing further questions. 
TURÁN PROBLEMS FOR INTEGER-WEIGHTED GRAPHS 3

\section{OVERVIEW OF RESULTS}

Since many problems in combinatorics have weighted versions it seems natural to study a weighted generalization of Erdös' problem. The case in which the edges can have nonnegative integer weight corresponds naturally to a multigraph question. We even allow negative (integer) multiplicities, when the solution proves to be simpler. In this section we collect some consequences of our main results.

Formally, an integer weighting of a graph $G$ is a function on its edges $w: E(G) \rightarrow \mathbb{Z}$. The weight of a subset of vertices $A$ is simply the total weight on all of its induced edges, $w(A):=\sum\{w(e): e \in E(H), e \subseteq A\}$. A weighted graph $(G, w)$ is $(k, r)$-dense if every set of $k$ vertices has weight at most $r$. The weighted Turán numbers are defined as

$$
\operatorname{ex}_{\mathbb{Z}}(n, k, r):=\max \{w(G):|V(G)|=n,(G, w) \text { is }(k, r) \text {-dense }\} .
$$

Since every non-edge can be considered to be an edge of weight 0 , it will suffice to consider $G=K_{n}$. We can refer to a graph by the corresponding $\{0,1\}$-weighting, where edges are weight 1 and non-edges are weight 0 . Multigraphs can also be viewed as weightings of $K_{n}$ with non-negative integer weights.

In this paper a simple method is given to determine $\operatorname{ex}_{\mathbb{Z}}(n, k, r)$ asymptotically for all positive $k$ and $r$. Define $m(k, r)$ as

$$
m(k, r):=\min \left\{m: \sum_{i<k}\lfloor 1+m i\rfloor>r\right\} .
$$

It is easy to see that $m$ is a rational number and for fixed $k$ it takes all non-negative rationals with denominator at most $k-1$. In Theorem 1 we obtain the following asymptotic

$$
\operatorname{ex}_{\mathbb{Z}}(n, k, r)=m(k, r)\left(\begin{array}{l}
n \\
2
\end{array}\right)+\mathrm{O}(n) .
$$

Furthermore, we give exact answers for $\operatorname{ex}_{\mathbb{Z}}(n, k, r)$ for at least half of the pairs $(k, r)$ for all $n$ in Theorem 3. Additional exact answers for some of the remaining cases can be found in Theorems 4, 6 and 8 .

We also provide asymptotic results in the case when the weights are taken from the set of natural numbers $\mathbb{N}=\{0,1,2,3, \ldots\}$. Let $\operatorname{ex}_{\mathbb{N}}(n, k, r)$ denote the maximum of $w(G)$ among all $n$-vertex $(k, r)$-dense graphs with non-negative weights (i.e., among all multigraphs without any specific bound on edge multiplicities). As a corollary to Theorem 9 we obtain that

$$
\operatorname{ex}_{\mathbb{N}}(n, k, r)=\operatorname{ex}_{\mathbb{Z}}(n, k, r)+\mathrm{O}(n)
$$

holds for $r \geq(k-1)\left(\begin{array}{l}k \\ 2\end{array}\right)$. Since $\operatorname{ex}_{\mathbb{Z}}(n, k, r)=\left(\begin{array}{c}n \\ 2\end{array}\right)+\operatorname{ex}_{\mathbb{Z}}\left(n, k, r-\left(\begin{array}{l}k \\ 2\end{array}\right)\right)$, we see that the determination of $\mathrm{ex}_{\mathbb{Z}}$ is necessary to solve the multigraph problem, ex $\mathbb{N}_{\mathbb{N}}$, for all $k$ and $r$.

We conclude this section by presenting a recent application of our results to a problem from the Turán theory for hypergraphs. Turán type problems for triple systems are rather 


\section{JOURNAL OF GRAPH THEORY}

difficult and only very few results are known. The intricacy of multigraphs lies between that of graphs and hypergraphs. Furthermore, it turns out that the study of Turán type problems for multigraphs has consequences for triple systems.

The Fano configuration $\mathcal{F}$, i.e., the projective plane of order two, is a hypergraph on 7 vertices and 7 triples where each pair of vertices is contained in a unique triple. The following triple-system contains no Fano plane: Partition the $n$-element underlying set into two almost equal sets. The hypergraph consisting of all $\frac{3}{4}\left(\begin{array}{l}n \\ 3\end{array}\right)+\mathrm{O}\left(n^{2}\right)$ triples meeting both sets cannot contain a Fano plane, since the Fano plane is not 2-colorable. The following old conjecture of Erdős and Sós is proved in [14]: Any set of $\frac{3}{4}\left(\begin{array}{l}n \\ 3\end{array}\right)+c n^{2}$ triples from an $n$-set, where $c$ is a suitable constant, must contain a copy of the Fano plane. Thus we obtain

$$
\operatorname{ex}(n, \mathcal{F})=\frac{3}{4}\left(\begin{array}{l}
n \\
3
\end{array}\right)+\mathrm{O}\left(n^{2}\right)
$$

The crucial fact used in [14] in the proof of (4) is that $\operatorname{ex}_{\mathbb{N}}(n, 4,20) \leq 3\left(\begin{array}{l}n \\ 2\end{array}\right)+\left\lfloor\frac{2}{3} n\right\rfloor$. This fact follows from our Theorem 8 , and from the observation that $\operatorname{ex}_{\mathbb{N}}(n, 4,20) \leq$ $\operatorname{ex}_{\mathbb{Z}}(n, 4,20)=3\left(\begin{array}{l}n \\ 2\end{array}\right)+\operatorname{ex}_{\mathbb{Z}}(n, 4,2)$.

\section{DENSITY RESULTS IN GRAPHS - A BRIEF HISTORY}

In this Section we survey a few basics of Turán theory to indicate the place of our results which we give in the next two Sections. We also use some part of the theory later in the proofs.

In 1963 Erdös [17] proposed the following problem: Given the parameters $n, k$ and $r$, determine the maximum number $\operatorname{ex}(n, k, r)$ of edges a graph $G$ on $n$ vertices can have if every $k$-vertex subgraph has at most $r$ edges. He reviewed all cases $k \leq 5$.

Let the Turán graph $T_{n, p}$ be the balanced complete $p$-partite graph on $n$ vertices, i.e., the $n$ vertices are grouped into $p$ parts as evenly as possible and two vertices are joined if and only if they belong to distinct parts. Let $t(n, p)$ denote the number of edges in $T_{n, p}$. We have $t(n, 2)=\left\lfloor n^{2} / 4\right\rfloor$, and in general

$$
t(n, p)=\left(1-\frac{1}{p}\right)\left(\begin{array}{l}
n \\
2
\end{array}\right)+\mathrm{O}(n) .
$$

Turán's theorem $[43,44]$ states that in the case $(k, r)=\left(k,\left(\begin{array}{l}k \\ 2\end{array}\right)-1\right)$, i.e., if $G$ does not contain a complete graph on $k$ vertices, $K_{k}$, as a subgraph then $G$ can have at most $t(n, k-1)$ edges. Further, the only graph having this many edges is $T_{n, k-1}$. The case $\operatorname{ex}(n, 3,2)=\left\lfloor n^{2} / 4\right\rfloor$ was proved earlier by Mantel [36]. Dirac [16] generalized Turán's theorem, for $r=\left(\begin{array}{l}k \\ 2\end{array}\right)-t$ with $1 \leq t \leq k / 2$ :

$$
\operatorname{ex}\left(n, k,\left(\begin{array}{l}
k \\
2
\end{array}\right)-t\right)=t(n, k-t)
$$


The question of Erdös is still unanswered, but one can briefly summarize the main results as follows. There are three types of answers, Gol'berg and Gurvich [27] call them zones. The linear zone consists of pairs $U^{1}:=\{(k, r): 0 \leq r<k-1\}$, the polynomial zone $U^{2}:=\left\{(k, r): k-1 \leq r<\left\lfloor k^{2} / 4\right\rfloor\right\}$ and finally $U^{3}:=\left\{(k, r):\left\lfloor k^{2} / 4\right\rfloor \leq r<\left(\begin{array}{l}k \\ 2\end{array}\right)\right\}$ is called the quadratic zone.

Trivially, $\operatorname{ex}(n, k, r)=r$ for $0 \leq r<(k-1) / 2$. For $0 \leq r<k-1$ we have

$$
\operatorname{ex}(n, k, r)=v-1+\left\lfloor\frac{u-1}{u}(x-v)\right\rfloor
$$

where $u=\lfloor(k-1) /(k-r-1)\rfloor$ and $v=k-1-u(k-r-2)$. We have $1 \leq u \leq v \leq r+1$. Here an example of an extremal $(k, r)$-dense graph is the disjoint union of one star on $v$ vertices and as many stars on $u$ vertices as possible, plus one remainder star on at most $u$ vertices. Note that (5) covers the case $0 \leq r<(k-1) / 2$ too, since in this case $u=1$.

In the polynomial range for $(k, r) \in U^{2}$ we have

$$
\Omega\left(n^{1+1 / k+1}\right) \leq \operatorname{ex}(n, k, r) \leq \mathrm{O}\left(n^{2-1 / k}\right)
$$

As Erdős [17] already pointed out, determining $\operatorname{ex}(n, k, k-1)$ is in fact equivalent to determining $\operatorname{ex}\left(n, C_{2 t}\right)$, the maximum number of edges of an $n$-vertex graph containing no cycle of length $2 t$, where $t=\lfloor k / 2\rfloor$. This is again basically equivalent to the problem of determining $\operatorname{ex}\left(n,\left\{C_{3}, C_{4}, \ldots, C_{2 t}\right\}\right)$, the maximum number of edges of an $n$ vertex graph of girth at least $2 t+1$. The upper bound of Bondy and $\operatorname{Simonovits~[4]~} \operatorname{states} \operatorname{ex}\left(n, C_{2 t}\right)=$ $\mathrm{O}\left(n^{1+\frac{1}{t}}\right)$. This is conjectured to give the correct order of magnitude. However, this is supported by a construction only for $t=2,3$ and 5 (see, e.g., Wenger [45], or Bollobás [3]). Erdős gave a probabilistic lower bound $\operatorname{ex}\left(n, C_{2 t}\right)>\Omega\left(n^{1+1 / 2 t}\right)$. In the remaining cases the best lower bound is given by the so-called "Ramanujan graphs" of Lubotzky, Phillips and Sarnak [35], Margulis [37] and Imrich [31]. Their construction was slightly improved by Lazebnik, Ustimenko and Woldar [34], who used ideas from the theory of Lie algebras to prove that $c n^{1+\frac{2}{3 t-2}}<\operatorname{ex}\left(n, C_{2 t}\right)$.

On the other hand, in the polynomial range a result of Kövári, T. Sós and Turán [32] implies that $\operatorname{ex}\left(n, k,\left\lfloor k^{2} / 4\right\rfloor-1\right) \leq \operatorname{ex}\left(n, K_{\lfloor k / 2\rfloor,\lceil k / 2\rceil}\right)=\mathrm{O}\left(n^{2-2 / k}\right)$.

Finally, in the quadratic zone, the Erdös-Stone-Simonovits $[22,23]$ theorem implies that

$$
\operatorname{ex}(n, k, r)=\left(1-\frac{1}{q}\right)\left(\begin{array}{l}
n \\
2
\end{array}\right)+o\left(n^{2}\right), \text { for } t(k, q) \leq r<t(k, q+1)
$$

Even more, it was recently summarized by Griggs, Simonovits, Thomas [28] (in a crude form also in Gol'berg and Gurvich [27]) that for each $q$ the range in the above inequality can be again cut into three parts where the error term is constant, linear, or polynomial. 
Let $r=t(k, q)+a($ where $0 \leq a<t(k, q+1)-t(k, q))$. Then for $n>n_{0}(k)$

$$
\operatorname{ex}(n, k, r)= \begin{cases}t(n, q)+a, & \text { for } 0 \leq a<\left\lfloor\frac{1}{2}\left\lceil\frac{k}{q}\right\rceil\right\rfloor \\ t(n, q)+\Theta(n), & \text { for }\left\lfloor\frac{1}{2}\left\lceil\frac{k}{q}\right\rceil\right\rfloor \leq a \leq\left\lceil\frac{k}{q}\right\rceil-2 \\ t(n, q)+\mathrm{o}\left(n^{2}\right), & \text { for all other } a \text { 's. }\end{cases}
$$

They also present a method to determine the linear error term precisely, and in the third zone the error term is super-linear. An interesting example of their results for this case is the description of the maximal graph for $k=6, r=11$. It is obtained from a complete bipartite graph $K[A, B]$ where the parts $A$ and $B$ do not necessarily have equal sizes, but $|A|,|B|=\frac{1}{2} n+\mathrm{o}(n)$ and one can add an arbitrary graph of girth 5 on $A$. Obviously, this example carries at most 11 edges in every 6 vertices, and it has $\frac{1}{4} n^{2}+\Theta\left(n^{3 / 2}\right)$ edges. (For $C_{4}$-free graphs see Brown [6] and Erdős, Rényi and Sós [21].)

The density problem $\operatorname{ex}(n, k, r)$ was investigated in several more papers. For example, Erdős, Faudree, Jagota and Euczak [19] deal with the case when $k=\Theta(n)$ and $r=\mathrm{O}\left(n^{2}\right)$.

A strongly related problem, the restricted multicoloring of the edges of the complete graph $K_{n}$, was investigated by Ahlswede, Cai and Zhang [1]. The resulting formulas are very similar. For recent developments see Axenovich and Kündgen [2].

Density problems in graphs are often difficult. Brown, Erdös and Sós [11, 12] proposed an even more difficult version - they investigated $h$-uniform hypergraphs, i.e., families of $h$-element sets. They introduced $\operatorname{ex}_{h}(n, k, r)$, the maximum number of edges in an $h$-uniform hypergraph of order $n$ such that no $k$ vertices span more than $r$ edges. For example, a $(4,2)$-free family of triples is a (partial) Steiner family, we have $\operatorname{ex}_{3}(n, 4,1) \leq$ $n(n-1) / 6$. Also see Gurvich [29].

A celebrated result of Ruzsa and Szemerédi [40] deals with (6,3)-free triple systems

$$
\operatorname{ex}_{3}(n, 6,2)=\mathrm{o}\left(n^{2}\right) \quad \text { but } \quad \lim _{n \rightarrow \infty} \operatorname{ex}_{3}(n, 6,2) / n^{2-c}=\infty \text { for all } c>0
$$

Some generalizations and a proof involving Szemerédi's regularity lemma can be found in Erdős, Frankl, Rödl [20].

\section{THE GENERALIZED DENSITY PROBLEM}

Definition. A weighting of a graph $G$ from a set of weights $W \subset \mathbb{R}$ is a function $w: E(G) \rightarrow W$ that assigns a weight to each edge. The weight of a subgraph $H$ of $G$ is just the total weight on all of its edges, that is

$$
w(H):=\sum\{w(e): e \in E(H)\} .
$$

The weight of a vertex set $A$ is the weight of the subgraph induced by $A, w(A):=w\left(\left.G\right|_{A}\right)$. For example for every vertex $v, w(\{v\})=0=w(\emptyset)$. We will also define the weight of a vertex $v$ by $w(v):=\sum\{w(u v): u v \in E(G)\}$, but we will only use this notion in 


\section{TURÁN PROBLEMS FOR INTEGER-WEIGHTED GRAPHS 7}

Sections 9. and 10. A weighted graph $(G, w)$ is $(k, r)$-dense if every set of $k$ vertices has weight at most $r$. In this language we define the weighted Turán numbers as

$$
\operatorname{ex}_{W}(n, k, r):=\sup \{w(G):|V(G)|=n,(G, w) \text { is }(k, r)-\text { dense }\} \text {. }
$$

Since every non-edge can be considered to be an edge of weight 0 , we will always assume that $0 \in W$, so that it will suffice to consider $G=K_{n}$. From now on, when we refer to a graph we mean the corresponding $\{0,1\}$-weighting, where edges are weight 1 and non-edges are weight 0 . Multigraphs can be viewed as $\mathbb{N}$-weightings of $K_{n}$, where $\mathbb{N}=\{0,1,2,3, \ldots\}$.

The problem of determining $\operatorname{ex}_{W}(n, k, r)$ for various choices of $W \subset \mathbb{R}$ has already been implicitly suggested in earlier work of Brown, Erdős and Simonovits $[7,8,9,10]$. The special case $W=\mathbb{Z}$, which we will extensively study in this paper, has been recently studied by Bondy and Tuza [5], and Kuchenbrod [33].

Bondy and Tuza show that in the Turán case negative weights don't help:

$$
\operatorname{ex}_{\mathbb{Z}}\left(n, k,\left(\begin{array}{l}
k \\
2
\end{array}\right)-1\right)=t(n, k-1)=\operatorname{ex}_{\{0,1\}}\left(n, k,\left(\begin{array}{l}
k \\
2
\end{array}\right)-1\right) .
$$

They prove that in this case the Turán graph is the (up to isomorphism) unique extremal weighting for $n \geq 2 k-2 \geq 6$, but that for $n=2 k-3 \geq 6$ there are exactly 5 non-isomorphic extremal weightings. They also determine all extremal weightings for $\operatorname{ex}_{\mathbb{Z}}(n, 3, r)$ : for example in the case $r=2$ the number of non-isomorphic extremal weightings is of order $c^{\sqrt{n}}$.

Kuchenbrod obtains results of the form $\operatorname{ex}_{\mathbb{Z}}(n, k, f(k))=f(n)$ for several functions $f$, among them $f(x)=t(x, s)(s \geq 1), f(x)=\left\lfloor\frac{k-2}{k-1} x\right\rfloor, f(x)=\left(x-1-\frac{s}{2}\left\lfloor\frac{x-1}{s}\right\rfloor\right)\left\lfloor\frac{x+s-1}{s}\right\rfloor$ $(3 \leq s \leq k-1)$ and $f(x)=\left\lfloor\frac{2 x+3}{5}\right\rfloor\left\lceil\frac{3 x+7}{5}\right\rceil-\left\lceil\left\lfloor\frac{2 x+3}{5}\right\rfloor^{2} / 4\right\rceil$. Using these results and solving several exceptional cases, he determines $\operatorname{ex}_{\mathbb{Z}}(n, k, r)$ exactly for all $k \leq 7$.

We will determine the exact value of $\operatorname{ex}_{\mathbb{Z}}(n, k, r)$ in many additional cases. We give a conjecture for its value in the remaining cases, which we can prove to be exact up to an additive error term of $n / 2$. Thus, asymptotically our answers are very sharp, since usually $\operatorname{ex}_{\mathbb{Z}}(n, k, r)=\Theta\left(n^{2}\right)$.

It is an easy observation that $\operatorname{ex}_{W}(n, k, r) /\left(\begin{array}{l}n \\ 2\end{array}\right)$ is a monotone non-increasing sequence in $n$, that is for $k \leq n^{\prime}<n$, we have

$$
\frac{\operatorname{ex}_{W}(n, k, r)}{\left(\begin{array}{l}
n \\
2
\end{array}\right)} \leq \frac{\operatorname{ex}_{W}\left(n^{\prime}, k, r\right)}{\left(\begin{array}{c}
n^{\prime} \\
2
\end{array}\right)}
$$

Indeed, for any $(k, r)$-dense weighting $w$ of $K_{n}$, we have

$$
\frac{w\left(K_{n}\right)}{\left(\begin{array}{c}
n \\
2
\end{array}\right)}=\frac{w\left(K_{n}\right)\left(\begin{array}{c}
n-2 \\
n^{\prime}-2
\end{array}\right)}{\left(\begin{array}{c}
n^{\prime} \\
2
\end{array}\right)\left(\begin{array}{c}
n \\
n^{\prime}
\end{array}\right)}=\frac{\sum\left\{w(A):|A|=n^{\prime}, A \subset V\left(K_{n}\right)\right\}}{\left(\begin{array}{c}
n^{\prime} \\
2
\end{array}\right)\left(\begin{array}{c}
n \\
n^{\prime}
\end{array}\right)} \leq \frac{\operatorname{ex}_{W}\left(n^{\prime}, k, r\right)}{\left(\begin{array}{c}
n^{\prime} \\
2
\end{array}\right)} .
$$


Hence the asymptotic density

$$
\operatorname{ex}_{W}(k, r):=\lim _{n \rightarrow \infty} \frac{\operatorname{ex}_{W}(n, k, r)}{\left(\begin{array}{l}
n \\
2
\end{array}\right)}
$$

exists for all $k$ and $r \geq 0$. From the monotonicity we also get the counting bound

$$
\operatorname{ex}_{W}(n, k, r) \leq \frac{\operatorname{ex}_{W}(k, k, r)}{\left(\begin{array}{l}
k \\
2
\end{array}\right)}\left(\begin{array}{l}
n \\
2
\end{array}\right) \leq \frac{r}{\left(\begin{array}{l}
k \\
2
\end{array}\right)}\left(\begin{array}{l}
n \\
2
\end{array}\right)
$$

If $W$ is dense in $\mathbb{R}$ then the counting bound is always achieved, equalities hold in (8). We focus our interest on the case when all weights as well as $r$ are integers. For example, from the reformulation of the Erdös-Stone-Simonovits theorem, (6), we obtain

$$
\operatorname{ex}_{\{0,1\}}(k, r)=1-\frac{1}{q}, \text { for } t(k, q) \leq r<t(k, q+1) .
$$

Thus the possible limit points form a well-ordered set under the usual ordering of the reals when $W=\{0,1\}$. Recall that a set is well-ordered with respect to a linear ordering if every non-empty subset has a least element, or equivalently if every nonmaximal element has a unique successor. Brown, Erdős and Simonovits $[7,8]$ proved that the same is true in the case of multigraphs with maximum edge multiplicity 2, i.e. $W=\{0,1,2\}$, (and also for digraphs $[9,10]$ ). However, more recent results of Rödl and Sidorenko [39] about the Turán number of multigraphs with edge multiplicities 4 or more state that in general the limit points of $\operatorname{ex}_{[m]}(n, G) /\left(\begin{array}{c}n \\ 2\end{array}\right)$ are not well-ordered. This makes it very unlikely that the set $\operatorname{ex}_{W}(k, r)$ is well-ordered for $W=[m]:=\{0,1,2, \ldots, m\}$ for any given $m \geq 4$, but this problem and many others remain open.

Our main result in this paper is to determine $\operatorname{ex}_{\mathbb{Z}}(k, r)$ for all $(k, r)$. For fixed $k$ it takes all non-negative rationals with denominator at most $k-1$. Furthermore, we will give constructions of graphs with positive and negative integer weights (i.e., functions $\left.w: E\left(K_{n}\right) \rightarrow \mathbb{Z}\right)$ and show that these constructions give the exact answer for $\operatorname{ex}_{\mathbb{Z}}(n, k, r)$ for at least half the pairs $(k, r)$. We also show that they are asymptotically optimal and, in contrast to $(6)$, our error term is always at most $\mathrm{O}(n)$.

We apply our results to determine $\operatorname{ex}_{\mathbb{N}}(k, r)$ for infinitely many pairs $(k, r)$. The study of $\operatorname{ex}_{\mathbb{N}}(n, k, r)$, i.e., to study multigraphs without upper bound on the multiplicities on the edges, was proposed by de Caen and Székely [15], who needed the case $(k, r)=(4,15)$. However the problem in general is more difficult and several cases remain open. The case $W=\mathbb{N}$ is particularly interesting, since it is a generalization of Turán's theorem for multigraphs.

\section{MAIN RESULTS}

Let $\mathbb{N}=\{0,1,2,3 \ldots\}$ denote the non-negative integers. For a positive integer $k$ and a real $m \geq 0$ let $R_{k}(m)$ be the closed region in $\mathbb{R}^{2}$ bounded by the inequalities $1 \leq x \leq k-1$ 


\section{TURÁN PROBLEMS FOR INTEGER-WEIGHTED GRAPHS 9}

and $0 \leq y \leq m x$ (See Figure 1). This region contains exactly $\left|R_{k}(m) \cap \mathbb{Z}^{2}\right|=\sum_{i<k}\lfloor 1+$ $m i\rfloor$ integer lattice points. We will define $m(k, r)$ to be the smallest slope $m$ so that $R_{k}(m)$ contains at least $r+1$ lattice points,

$$
m(k, r):=\min \left\{m:\left|R_{k}(m) \cap \mathbb{Z}^{2}\right|>r\right\} .
$$

For example, in the case $k=11, r=32$ the region $R_{11}(1 / 2)$ in Figure 1 contains 35 lattice points, 5 of them on its upper edge, showing that $m(11,30)=m(11,31)=\cdots=$ $m(11,34)=1 / 2$.

Our main result is that the slope $m$ obtained from (9) yields the asymptotic density in the case when $W=\mathbb{Z}$ :

$$
\operatorname{ex}_{\mathbb{Z}}(k, r)=m(k, r) .
$$

Moreover, the region $R_{n}(m)$ is intimately related to the maximal weight of a $(k, r)$-dense graph:

$$
0 \leq\left|R_{n}(m) \cap \mathbb{Z}^{2}\right|-\operatorname{ex}_{\mathbb{Z}}(n, k, r)<n
$$

holds for all $n \geq k$. Summarizing, we have

Theorem 1. (Asymptotic)

$$
\operatorname{ex}_{\mathbb{Z}}(n, k, r)=m(k, r)\left(\begin{array}{l}
n \\
2
\end{array}\right)+\mathrm{O}(n) .
$$

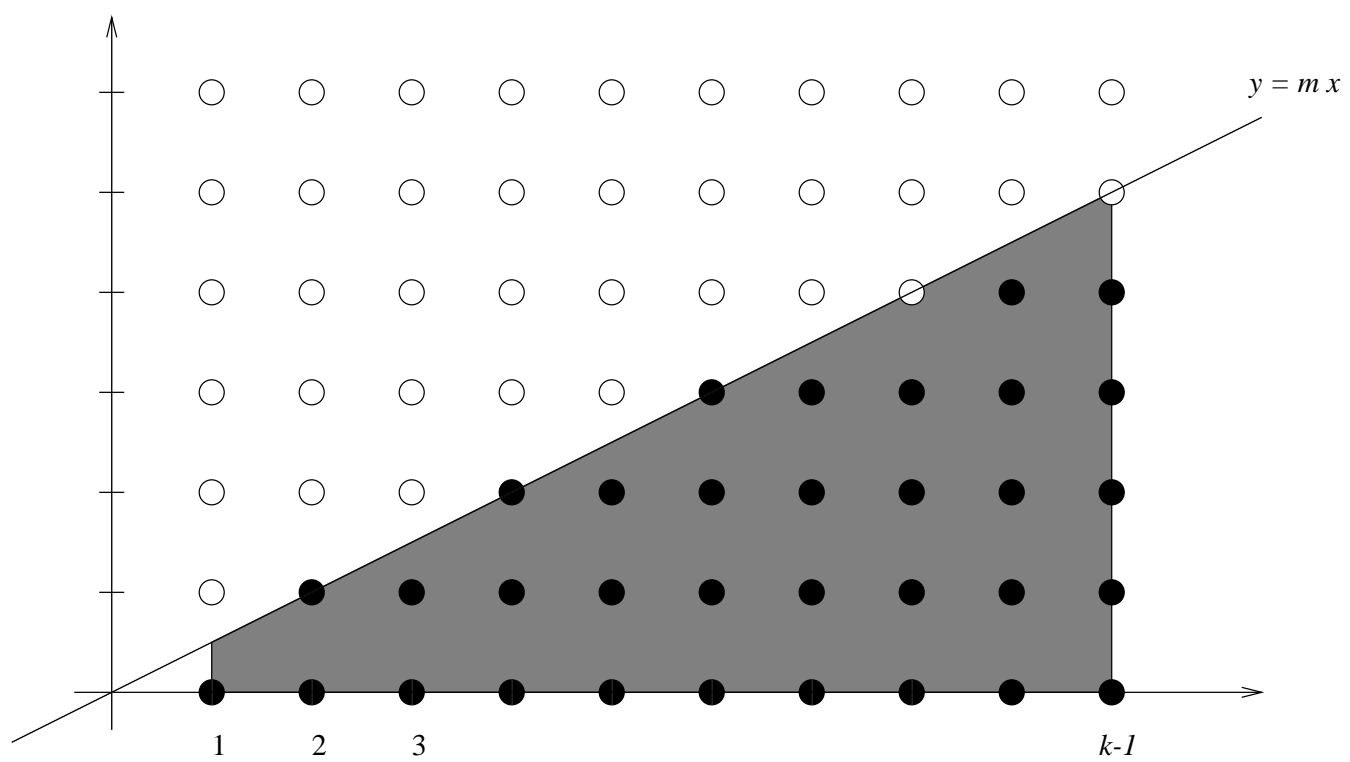

Figure 1: $R_{k}(m)$ with $(k, r)=(11,32)$. We have $\ell=5$ and $a=3$. 
In other words, if we put $r+1$ lattice points in the region $0<x<k, y \geq 0$, "as far down as possible", then the smallest slope $m$ of any line through the origin that creates a region containing all these points gives the asymptotic density. Obviously, $m$ is always a rational number and can be written in reduced form, $m=p / q$ with g.c.d. $(p, q)=1$ and $q>0$.

To further determine $\operatorname{ex}_{\mathbb{Z}}(n, k, r)$ let $\ell_{k}(p / q)$ denote the number of lattice points of $R_{k}(p / q)$ on its upper edge, $\ell_{k}(p / q)=\lfloor(k-1) / q\rfloor$. Place the $r+1$ lattice points into $R_{k}(p / q)$ by using all points below the line $y=\frac{p}{q} x$. Then let $a_{k}(r)$ denote the number of points we have to use on the upper boundary. We have

$$
1 \leq a_{k}(r)=r+1-\left(\left|R_{k}(p / q) \cap \mathbb{Z}^{2}\right|-\ell_{k}(p / q)\right)=r+1-\sum_{i<k}\left\lceil\frac{p}{q} i\right\rceil \leq \ell_{k}(p / q) .
$$

From now on, we abbreviate these two functions as $\ell$ and $a$.

In Section 6. we will give the upper bound for Theorem 1 by establishing

Theorem 2. (Upper bound for all values)

$$
\operatorname{ex}_{\mathbb{Z}}(n, k, r) \leq \sum_{i<n}\left\lceil\frac{p}{q} i\right\rceil+ \begin{cases}a-1, & \text { for } a \leq(\ell+1) / 2, \\ \left\lfloor\frac{n-1}{q}\right\rfloor+a-\ell-1, & \text { for } a>(\ell+1) / 2 .\end{cases}
$$

The proof of Theorem 2 essentially uses the monotonicity of the density (7) in the following form.

$$
\operatorname{ex}_{\mathbb{Z}}(n+1, k, r) \leq\left\lfloor\frac{\left(\begin{array}{c}
n+1 \\
2
\end{array}\right)}{\left(\begin{array}{c}
n \\
2
\end{array}\right)} \operatorname{ex}_{\mathbb{Z}}(n, k, r)\right\rfloor=\operatorname{ex}_{\mathbb{Z}}(n, k, r)+\left\lfloor\frac{2}{n-1} \operatorname{ex}_{\mathbb{Z}}(n, k, r)\right\rfloor
$$

In Section 7. we will give a recursive construction for the lower bound in Theorem 1. Furthermore, we will find the exact value for many cases in Section 8.:

Theorem 3. (Exact answer for half of the cases) If $2 a \leq \ell+1$, then

$$
\operatorname{ex}_{\mathbb{Z}}(n, k, r)=\sum_{i<n}\left\lceil\frac{p}{q} i\right\rceil+a-1 .
$$

Theorem 4. (Exact answer for small $n$ ) If $2 a \geq \ell+1$ and $n \leq 2 a q$, then

$$
\operatorname{ex}_{\mathbb{Z}}(n, k, r)=\sum_{i<n}\left\lceil\frac{p}{q} i\right\rceil+\left\lceil\frac{n}{q}\right\rceil+a-\ell-2 .
$$




\section{TURÁN PROBLEMS FOR INTEGER-WEIGHTED GRAPHS 11}

Note that $\left\lfloor\frac{n-1}{q}\right\rfloor=\left\lceil\frac{n}{q}\right\rceil-1$ so the upper bound for $\mathrm{ex}_{\mathbb{Z}}$ in Theorem 4 follows from Theorem 2. Theorem 4 gives exact answers only for small values of $n, n<2 k$, and in the case $a=(\ell+1) / 2$ its range is included in $\{n: k \leq n<k+q\}$. This is the reason that in this case the two kinds of remainders in Theorem 2 coincide.

In Section 9. we will establish the lower bound that we believe to be optimal.

Theorem 5. (Lower bound)

$$
\operatorname{ex}_{\mathbb{Z}}(n, k, r) \geq \sum_{i<n}\left\lceil\frac{p}{q} i\right\rceil+\operatorname{ex}_{\mathbb{Z}}(\lceil n / q\rceil, \ell+1, a-1) .
$$

Theorems 3 and 4 show that in the respective cases $2 a \leq \ell+1$ and $n \leq 2 a q$ equality holds. We conjecture that equality always holds, in other words that the linear term is itself of the form $\operatorname{ex}_{\mathbb{Z}}(n, k, r)$, for $r \leq k-2$. In Section 10. we give a conjecture for the linear term, which we can prove for large $n$ (see Theorem 6). Proving both conjectures would completely settle the problem in the case when we allow integer weights.

Note that for $m(k, r) \neq 0$ Theorem 1 yields the order of magnitude and the leading coefficient of $\operatorname{ex}_{\mathbb{Z}}(n, k, r)$ (it is $\left.(1 / 2) m(k, r) n^{2}+\mathrm{O}(n)\right)$ and it gives very tight bounds. For the missing case, $m(k, r)=0$, we have the next result.

Theorem 6. (Exact value for $m=0$ and large $n$ )

Suppose that $0<r \leq k-2$ and $n \geq 3 k r$. Then

$$
\operatorname{ex}_{\mathbb{Z}}(n, k, r)=r+\left\lfloor\frac{u-1}{u}(n-k+1)\right\rfloor
$$

where $u=\lfloor(k-1) /(k-1-r)\rfloor$. 


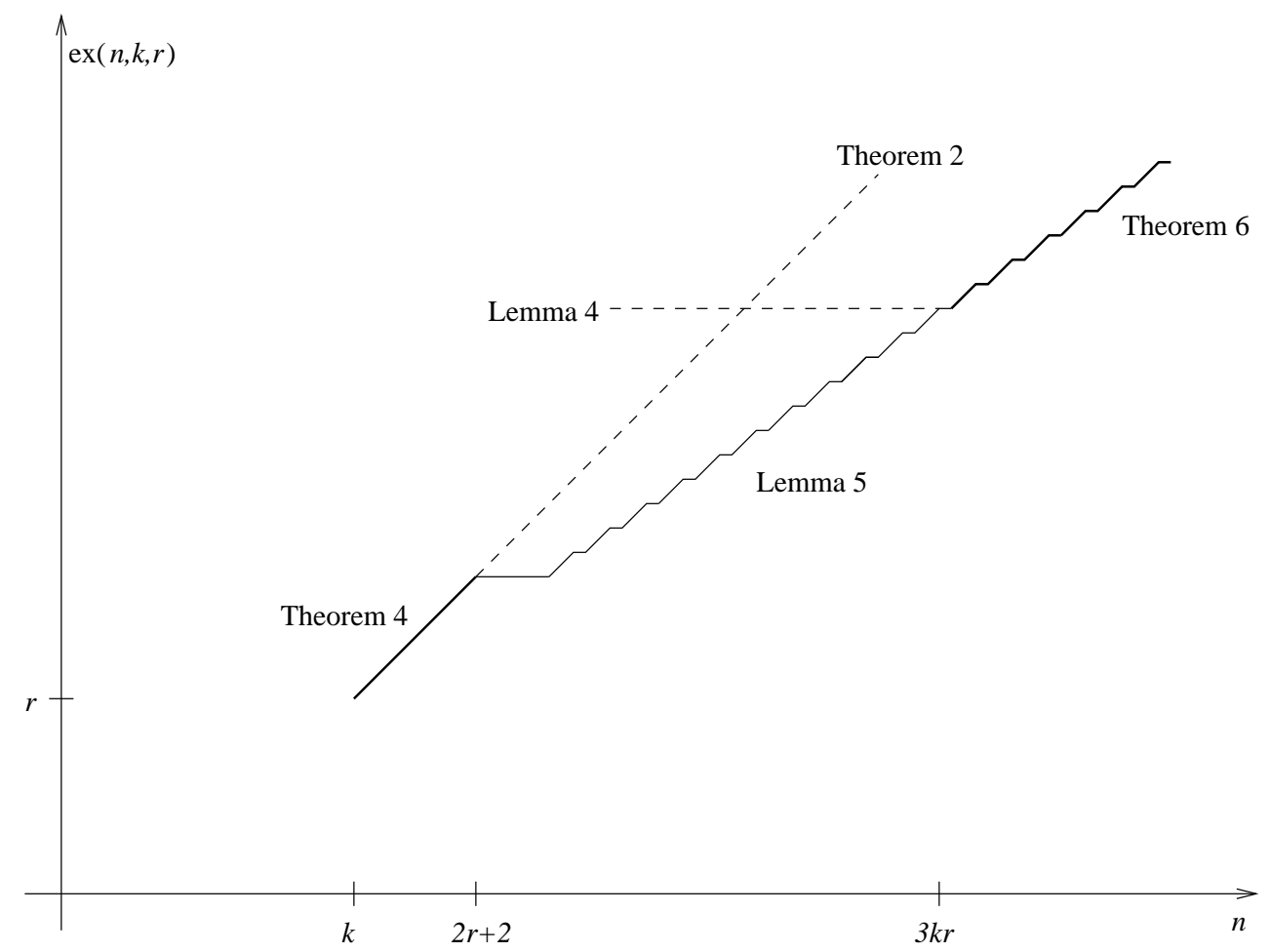

Figure 2: The linear case. $\quad k=12, r=8, u=3$.

Finally in Theorem 9 in Section 11. we address the multigraph case $W=\mathbb{N}$, by observing that many of the constructions previously given do not use negative weights and are therefore also optimal in this case.

\section{PROOF OF THE GENERAL UPPER BOUND}

In this Section we first study a recurrence relation. After that, the proof of Theorem 2 will be immediate. In the next statement let $p, q, a$ and $\ell$ as in Section 5 .

Theorem 7. Suppose that $f=f_{k, r}$ is an integer function defined by the starting value $f(k)=r \geq 0$ and for $n \geq k>0$ by the recursion $f(n+1)=\left\lfloor\frac{n+1}{n-1} f(n)\right\rfloor$. Then

$$
f(n)=\sum_{i<n}\left\lceil\frac{p}{q} i\right\rceil+ \begin{cases}a-1, & \text { for } a \leq(\ell+1) / 2, \\ \left\lfloor\frac{n-1}{q}\right\rfloor+a-\ell-1, & \text { for } a>(\ell+1) / 2 .\end{cases}
$$


This Theorem, in a slightly different form, is due to Gessel and Indik [26]. (We are greatful to M. Simonovits for this information.) Our proof here is slightly more straightforward. Special cases appear in the literature in several places, even in papers related to Turán theory (e.g., Ahlswede, Cai, Zhang [1], Stechkin [41] and Stechkin, Frankl [42]). Frequently it is stated and used in the form of inequalities, i.e., if for some sequence of integers $s_{k}, s_{k+1}, \ldots$ one has

$$
s_{k}=r \text { and } \frac{s_{n+1}}{\left(\begin{array}{c}
n+1 \\
2
\end{array}\right)} \leq \frac{s_{n}}{\left(\begin{array}{c}
n \\
2
\end{array}\right)} \text { for } n \geq k, \text { then } s_{n} \leq f_{k, r}(n)
$$

holds. For example the case $k=5, r=7$ (i.e., $\left.f_{5,7}(n)=\left(n^{2}+n\right) / 2\right)$ was proved in [24]. Guy [30] also suggested that these and similar recurrences may be worth studying for their own sake.

Proof of Theorem \%. We will proceed by induction on $n$. Write $f(n)$ in the form $\sum_{i=1}^{n-1}\left\lceil\frac{p}{q} i\right\rceil+h(n)$. It suffices to establish

$$
\left\lfloor\frac{2}{n-1} f(n)\right\rfloor=\left\lceil\frac{p}{q} n\right\rceil+\varepsilon
$$

where $\varepsilon=1$ if $q$ divides $n$ and $2 h(n) \geq\lfloor(n-1) / q\rfloor$ and $\varepsilon=0$ otherwise. Simultaneously to (13) we use induction to prove that

$$
0 \leq h(n)<\lfloor(n-1) / q\rfloor
$$

We establish the following flow of implications (Theorem 7 for $f(n)) \Longrightarrow((14)$ for $h(n)$ ) $\Longrightarrow((13)$ for $f(n)) \Longrightarrow$ (Theorem 7 for $f(n+1))$. Here the first implication is obvious. For the base case $n=k$ we have

$$
f(k)=r=\sum_{i<k}\left\lceil\frac{p}{q} i\right\rceil+a-1=\sum_{i<k}\left\lceil\frac{p}{q} i\right\rceil+a-1+\left\lfloor\frac{k-1}{q}\right\rfloor-\ell,
$$

and thus (14) holds for $h(k)$.

Next we consider $2 f(n) /(n-1)$. Observe that

$$
2 f(n)=\sum_{i=1}^{n-1}\left(\left\lceil\frac{p}{q} i\right\rceil+\left\lceil\frac{p}{q}(n-i)\right\rceil\right)+2 h \geq(n-1)\left\lceil\frac{p}{q} n\right\rceil
$$

In the case when $q$ does not divide $n$ we also conclude that

$$
\sum_{i=1}^{n-1}\left(\left\lceil\frac{p}{q} i\right\rceil+\left\lceil\frac{p}{q}(n-i)\right\rceil\right)+2 h \leq(n-1)\left(\left\lceil\frac{p}{q} n\right\rceil+1\right)-2\lfloor(n-1) / q\rfloor+2 h .
$$


This inequality holds since for $i$ or $n-i$ divisible by $q$ we can drop the corresponding ceiling and save one. Thus

$$
\left\lceil\frac{p}{q} n\right\rceil \leq\left\lfloor\frac{2}{n-1} f(n)\right\rfloor \leq\left\lceil\frac{p}{q} n\right\rceil+\left\lfloor 1+2 \frac{h-\lfloor(n-1) / q\rfloor}{n-1}\right\rfloor \leq\left\lceil\frac{p}{q} n\right\rceil .
$$

In the case when $q$ does divide $n$ we get that

$$
2 f(n)=(n-1)\left(\left\lceil\frac{p}{q} n\right\rceil+1\right)-\lfloor(n-1) / q\rfloor+2 h,
$$

so that for $2 h<\lfloor(n-1) / q\rfloor$ again

$$
\left\lfloor\frac{2}{n-1} f(n)\right\rfloor=\left\lceil\frac{p}{q} n\right\rceil+\left\lfloor 1+\frac{2 h-\lfloor(n-1) / q\rfloor}{n-1}\right\rfloor=\left\lceil\frac{p}{q} n\right\rceil,
$$

and for $2 h \geq\lfloor(n-1) / q\rfloor$

$$
\left\lfloor\frac{2}{n-1} f(n)\right\rfloor=\left\lceil\frac{p}{q} n\right\rceil+\left\lfloor 1+\frac{2 h-\lfloor(n-1) / q\rfloor}{n-1}\right\rfloor=\left\lceil\frac{p}{q} n\right\rceil+1 .
$$

In the case $a \leq(\ell+1) / 2$ we have $2 h=2 a-2 \leq \ell-1=\lfloor(k-1) / q\rfloor-1<\lfloor(n-1) / q\rfloor$ so that $\varepsilon=0$ for all $n$. Thus $h(n)=a-1$ for every $n \geq k$.

In the case $a>(\ell+1) / 2$ we have $2 h(k)>\lfloor(k-1) / q\rfloor$, and by induction we obtain that $2 h(n)>\lfloor(n-1) / q\rfloor$, because $\lfloor(n-1) / q\rfloor+\varepsilon=\lfloor n / q\rfloor$, since $\varepsilon=0$ unless $n / q$ is an integer.

Proof of Theorem 2. This is immediate from Theorem 7. For the base case we have $\operatorname{ex}_{\mathbb{Z}}(k, k, r) \leq r=f_{k, r}(r)$. Then $\operatorname{ex}_{\mathbb{Z}}(n, k, r) \leq f(n)$ is implied by the monotonicity of $\operatorname{ex}_{W}(n, k, r) /\left(\begin{array}{l}n \\ 2\end{array}\right)$, see $(12)$.

Unfortunately, this upper bound does not always give the exact value of $\operatorname{ex}_{\mathbb{Z}}(n, k, r)$.

\section{CONSTRUCTION OF DENSE GRAPHS FOR INTEGER WEIGHTS}

In this section a recursive construction is given which yields the right lower bound up to a $\mathrm{O}(n)$ error term. To get the best possible result we formulate it in a slightly more general way. One of our technical difficulties is that we do not $\mathrm{know} \operatorname{ex}_{\mathbb{Z}}(n, k, r)$ for all $n>k \geq r+2 \geq 2$ (i.e., when it is linear in $n$ according to Theorem 6), so we are going to introduce a linear error term function $h$.

The main idea is to obtain a structure similar to that of $T_{n, k}$ with the right properties. We distribute the vertices as equally as possible into $q$ classes. The weights on the edges are determined by a $q$ by $q$ matrix $M(p / q)$, that is $w(u v)=M_{i, j}$ if $u$ is in the $i$-th class 
and $v$ in the $j$-th. In the Turán case $T_{n, k}, M=M((k-1) / k)$ is the $k$ by $k$ matrix with all entries 1 except the main diagonal which consists of zeros. Other examples for $M(p / q)$ are in Figure 3.

$$
[0] \quad\left[\begin{array}{ccc}
-1 & 1 & 1 \\
1 & 0 & 0 \\
1 & 0 & 0
\end{array}\right]\left[\begin{array}{cccccccc}
-2 & 0 & 0 & 1 & 1 & 1 & 1 & 1 \\
0 & -1 & -1 & 1 & 1 & 1 & 1 & 1 \\
0 & -1 & -1 & 1 & 1 & 1 & 1 & 1 \\
1 & 1 & 1 & 0 & 0 & 0 & 0 & 0 \\
1 & 1 & 1 & 0 & 0 & 0 & 0 & 0 \\
1 & 1 & 1 & 0 & 0 & 0 & 0 & 0 \\
1 & 1 & 1 & 0 & 0 & 0 & 0 & 0 \\
1 & 1 & 1 & 0 & 0 & 0 & 0 & 0
\end{array}\right]
$$

Figure 3: $M(0 / 1), M(1 / 3)$ and $M(3 / 8)$.

These constructions will asymptotically yield the correct density $p / q$. However in some cases we can improve the construction, by adding a small forest-like construction to the class with the highest negative weight, that is in Figure 3 in the top left corner. This will result in adding a linear number of edges.

To capture the behavior of this linear term we will need the following

Definition. A function $h: \mathbb{N} \rightarrow \mathbb{N}$ is called a linear weighting function if it has the following properties:

(L1) Monotonicity: $h(x) \leq h(x+1)$,

(L2) Lipschitz: $h(x+1) \leq h(x)+1$, and

(L3) For every $n \in \mathbb{N}$ there exists a weighting $w$ of $K_{n}$ with integer weights such that $w\left(K_{n}\right)=h(n)$ and $w(A) \leq h(|A|)$ for every vertex set $A$.

Notice that this forces $h(1)=0=h(0)$ so that we only need to specify $h$ for $x>1$. The zero function is an example of a linear weighting function and we will give other examples in Sections 8. and 9. We call these weighting functions linear since $h(n)=\mathrm{O}(n)$.

We will now give a recursive construction establishing

Lemma 1. For every linear weighting function with $h(\ell+1) \leq a-1$

$$
\operatorname{ex}_{\mathbb{Z}}(n, k, r) \geq \sum_{i<n}\left\lceil\frac{p}{q} i\right\rceil+h(\lceil n / q\rceil) .
$$

Using the zero function for $h$, which corresponds to the pure matrix construction without adding a linear term, we immediately obtain the lower bound for Theorem 1, 
finishing its proof. Notice that the difference between the lower bound from Lemma 1 and the upper bound in Theorem 2 is less than $n / q$. The reader may want to follow the proof in the matrix structure with the examples provided in Figure 3, assuming in the mean time that $h$ is the zero function.

Proof of Lemma 1. It suffices to consider the case where $0 \leq p<q$, since in general when $\frac{p}{q}=j+\frac{p^{\prime}}{q}$ and $r=a-1+\sum_{i<k}\left\lceil\frac{p}{q} i\right\rceil=a-1+j\left(\begin{array}{l}k \\ 2\end{array}\right)+\sum_{i<k}\left\lceil\frac{p^{\prime}}{q} i\right\rceil=r^{\prime}+j\left(\begin{array}{c}k \\ 2\end{array}\right)$, we get that

$$
\begin{aligned}
\operatorname{ex}_{\mathbb{Z}}(n, k, r) & =\operatorname{ex}_{\mathbb{Z}}\left(n, k, r^{\prime}+j\left(\begin{array}{l}
k \\
2
\end{array}\right)\right)=\operatorname{ex}_{\mathbb{Z}}\left(n, k, r^{\prime}\right)+j\left(\begin{array}{l}
n \\
2
\end{array}\right) \\
& \geq \sum_{i<n}\left\lceil\frac{p^{\prime}}{q} i\right\rceil+h(\lceil n / q\rceil)+j\left(\begin{array}{l}
n \\
2
\end{array}\right)=\sum_{i<n}\left\lceil\frac{p}{q} i\right\rceil+h(\lceil n / q\rceil) .
\end{aligned}
$$

We will also use the fact that $\lceil x\rceil=\lceil\lceil x q\rceil / q\rceil$, for $x \in \mathbb{R}$ and $q$ a positive integer.

Our recursive construction of a weight function $w$ for $K_{n}$ will satisfy the requirement that for every set $A$ of $z$ vertices $(0 \leq z \leq n)$ we get $w(A) \leq \sum_{i<z}\left\lceil\frac{p}{q} i\right\rceil+h(\lceil z / q\rceil)$, with equality for $z=n$. Hence the fact that $w$ is indeed $(k, r)$-dense immediately follows by considering the case $z=k$ :

$$
\left.\sum_{i<k}\left\lceil\frac{p}{q}\right\rceil\right\rceil+h(\lceil k / q\rceil)=(r-a+1)+h(\ell+1) \leq r
$$

We build the construction according to the size of the denominator $q$. In the base case $q=1$, we get that $p=0$, so that it suffices to give a weighting with $w(A) \leq h(z)$ and equality for $z=n$, but such a weighting is guaranteed by the definition of $h$. The corresponding matrix is $M(0 / 1)$.

For $q>1$ we get that $p>0$ since g.c.d. $(q, 0)=q>1$. So let $q=p(t+2)-p^{\prime}$ with $0 \leq p^{\prime}<p$ and $0 \leq t=\left\lceil\frac{q}{p}\right\rceil-2$. Let $n^{\prime}=\left\lceil\frac{n p}{q}\right\rceil$. Since g.c.d. $\left(p, p^{\prime}\right)=1$ we can start with a weight function $w^{\prime}$ for $K_{n^{\prime}}$ with $w^{\prime}(A) \leq \sum_{i<z}\left\lceil\frac{p^{\prime}}{p} i\right\rceil+h(\lceil z / p\rceil)$ for all $z$-sets $A$ and equality for $z=n^{\prime}$. From this we obtain the required weight function $w$ on $K_{n}$, by partitioning $V\left(K_{n}\right)=V\left(K_{n^{\prime}}\right) \cup V^{\prime}$ and defining

$$
w(u v)= \begin{cases}0 & u, v \in V^{\prime}, \\ 1 & u \in V^{\prime}, v \in V\left(K_{n^{\prime}}\right), \\ w^{\prime}(u v)-t & u, v \in V\left(K_{n^{\prime}}\right) .\end{cases}
$$

As an example $M(1 / 3)$ was constructed from $M(0 / 1)$ and $M(3 / 8)$ from $M(1 / 3)$ in this fashion, with $t=1$ in both cases.

To see that this works, take any $A \subset V\left(K_{n}\right)$ of size $z$ and let $\left|A \cap V\left(K_{n^{\prime}}\right)\right|=x$. We obtain 


$$
\begin{aligned}
w(A) & =x(z-x)-t\left(\begin{array}{l}
x \\
2
\end{array}\right)+w^{\prime}\left(A \cap V\left(K_{n^{\prime}}\right)\right) \\
& \leq x(z-x)-t\left(\begin{array}{l}
x \\
2
\end{array}\right)+\sum_{i=0}^{x-1}\left\lceil\frac{p^{\prime}}{p} i\right\rceil+h(\lceil x / p\rceil) \\
& =x(z-x)+2\left(\begin{array}{l}
x \\
2
\end{array}\right)+\sum_{i=0}^{x-1}\left\lceil-(t+2) i+\frac{p^{\prime}}{p} i\right\rceil+h(\lceil x / p\rceil) \\
& =x(z-1)+\sum_{i=0}^{x-1}\left\lceil-\frac{q}{p} i\right\rceil+h(\lceil x / p\rceil) \\
& =\sum_{i=0}^{x-1}\left\lceil z-1-\frac{q}{p} i\right\rceil+h(\lceil x / p\rceil) .
\end{aligned}
$$

Next we observe that

$$
\sum_{i=0}^{z-1}\left\lceil\frac{p}{q} i\right\rceil=\sum_{i=0}^{\left\lceil\frac{p}{q}(z-1)\right\rceil-1}\left\lceil z-1-\frac{q}{p} i\right\rceil,
$$

since both sums count the number of lattice points in $R_{z}(p / q)$ that are below the line $y=\frac{p}{q} x$, except that the first sum is counting vertical first, while the second one goes horizontal first. Furthermore $\left\lceil z-1-\frac{q}{p} i\right\rceil=0$ exactly when $\left\lceil\frac{p}{q}(z-1)\right\rceil \leq i \leq\left\lceil\frac{p}{q} z\right\rceil-1$. Hence if $\lceil z / q\rceil \geq\lceil x / p\rceil$, then by the monotonicity of $h$ and taking only the positive terms in the sum:

$$
w(A) \leq \sum_{i=0}^{\left\lceil\frac{p}{q}(z-1)\right\rceil-1}\left\lceil z-1-\frac{q}{p} i\right\rceil+h(\lceil z / q\rceil)=\sum_{i=0}^{z-1}\left\lceil\frac{p}{q} i\right\rceil+h(\lceil z / q\rceil) .
$$

If $\lceil z / q\rceil<\lceil x / p\rceil$, then $x>p\lceil z / q\rceil=p\lceil\lceil z p / q\rceil / p\rceil \geq\lceil z p / q\rceil$, so that

$$
\begin{aligned}
w(A) & \leq \sum_{i=0}^{\left\lceil\frac{p}{q}(z-1)\right\rceil-1}\left\lceil z-1-\frac{q}{p} i\right\rceil+\sum_{i=\left\lceil\frac{p}{q} z\right\rceil}^{x-1}\left\lceil z-1-\frac{q}{p} i\right\rceil+h(\lceil x / p\rceil) \\
& \leq \sum_{i=0}^{z-1}\left\lceil\frac{p}{q} i\right\rceil+\left(x-\left\lceil\frac{p}{q} z\right\rceil\right)(-1)+h(\lceil x / p\rceil) .
\end{aligned}
$$

Hence we are done if $x-\left\lceil\frac{p}{q} z\right\rceil \geq h(\lceil x / p\rceil)-h(\lceil z / q\rceil)$. However $h(\lceil x / p\rceil)-h(\lceil z / q\rceil) \leq$ $\left\lceil\frac{x}{p}\right\rceil-\left\lceil\frac{z}{q}\right\rceil$ by the Lipschitz property of $h$. So we obtain the desired result if $x-\left\lceil\frac{x}{p}\right\rceil \geq$ 
$\left\lceil\frac{p}{q} z\right\rceil-\left\lceil\frac{z}{q}\right\rceil=\left\lceil\frac{p}{q} z\right\rceil-\left\lceil\left\lceil\frac{z p}{q}\right\rceil / p\right\rceil$. This is true since the function $x-\left\lceil\frac{x}{p}\right\rceil$ is non-decreasing on integers and we assumed that $x>\lceil z p / q\rceil$.

Finally we need to check for equality when $z=n$. In that case $x=n^{\prime}=\left\lceil\frac{n p}{q}\right\rceil$ and thus, following the first chain of inequalities, we obtain:

$$
\begin{aligned}
w\left(K_{n}\right) & =\sum_{i=0}^{n^{\prime}-1}\left\lceil n-1-\frac{q}{p} i\right\rceil+h\left(\left\lceil n^{\prime} / p\right\rceil\right) \\
& \left.=\sum_{i=0}^{\lceil n p / q\rceil-1}\left\lceil n-1-\frac{q}{p} i\right\rceil+h(\lceil\lceil n p / q\rceil / p\rceil)\right) \\
& =\sum_{i=0}^{\left\lceil\frac{p}{q}(n-1)\right\rceil-1}\left\lceil n-1-\frac{q}{p} i\right\rceil+h(\lceil n / q\rceil) \\
& =\sum_{i=0}^{n-1}\left\lceil\frac{p}{q} i\right\rceil+h(\lceil n / q\rceil) .
\end{aligned}
$$

This finishes the proof of Lemma 1.

The construction yields the Turán graph $T_{n, q}$ when $p=q-1$. For $p=1$ we get a graph that is almost bipartite: $V=V_{1} \cup V_{2},\left|V_{1}\right|=\lceil n / q\rceil$ and all $V_{1} V_{2}$ edges have weight 1 , all edges within $V_{2}$ have weight 0 and all edges within $V_{1}$ have weight $2-q$, as indicated for example by $M(1 / 3)$. The constructions given by Figure 3 are all optimal in many cases. For example from Theorem 3 we will see that $M(3 / 8)$ yields an optimal construction when $(k, r)=(9,17),(10,21),(11,25),(12,30), \ldots$

\section{SOME EXACT VALUES OF $\operatorname{ex}_{\mathbb{Z}}(n, k, r)$}

Recall that a star is a graph $K_{1, n}$ where we will allow $n \geq 0$ in the context of this paper. For each star we call the vertex of degree $n$ its center, the other vertices are the leafs. If $n=1$ we designate only one of the two vertices to be the center and the other to be the leaf.

So far the zero function is the only linear weighting function we have seen. In this section we will explore other linear weighting functions, thus obtaining quick proofs for Theorems 3 and 4 . Consider $h_{a}(x)=\min \{x-1, a-1\}$. The first two properties (L1) and (L2) of a linear weighting function are easily checked and the required weighting is given by a star on $\min \{x, a\}$ vertices, with the remaining vertices being isolated. Notice that $h_{1}$ is the zero function.

Proof of Theorem 3. Using $h=h_{a}$ the lower bound is immediate from Lemma 1, since $a-1 \geq h\left(\left\lceil\frac{n}{q}\right\rceil\right) \geq h\left(\left\lceil\frac{k}{q}\right\rceil\right)=h(\ell+1) \geq h(a)=a-1$. 
The upper bound for $\operatorname{ex}_{\mathbb{Z}}(n, k, r)$ follows from Theorem 2 .

In the case when $a \leq \ell \leq 2 a-1$ we let

$$
h_{a, \ell}(x)= \begin{cases}x-1, & \text { for } x \leq a \\ a-1, & \text { for } a \leq x \leq \ell+1 \\ x+a-\ell-2, & \text { for } \ell+1 \leq x \leq 2 a \\ 3 a-\ell-2, & \text { for } 2 a \leq x\end{cases}
$$

So $h_{a, \ell}(x)=h_{a}(x)$ for $x \leq \ell+1$ and $h_{a, \ell}(x)=2 a-\ell-1+h_{a}(x-a)$ for $x \geq \ell+1$. Since the monotonicity and Lipschitz property of $h_{a, \ell}$ are again straight forward we only have to verify condition (L3) to see that $h_{a, \ell}$ is a linear weighting function. For this we will use two stars on $a$ vertices and connect their centers by an edge of weight $a-\ell \leq 0$. For $x>2 a$ the remaining vertices are isolated, and for $x<2 a$ we take the obvious subgraph. It can be seen that this satisfies condition (L3).

Proof of Theorem 4. The upper bound is exactly Theorem 2. The lower bound follows from Lemma 1 with $h=h_{a, \ell}$. It suffices to notice that $h_{a, \ell}(\ell+1)=a-1$ and that $2 a \geq\lceil n / q\rceil \geq\lceil k / q\rceil=\ell+1$.

\section{PROOF OF THE LOWER BOUND}

In this section we prove Theorem 5. We use the construction of Section 7. to obtain a probably optimal lower bound. To be able to apply Lemma 1 we show that for fixed values of $r$ and $k$, with $0 \leq r \leq k-2, \operatorname{ex}_{\mathbb{Z}}(x, k, r)$ is a linear weighting function. However $\operatorname{ex}_{\mathbb{Z}}(x, k, r)$ is only defined for $x \geq k$, hence we will let

$$
h(x)=h(x, k, r)= \begin{cases}x-1, & \text { for } x \leq r+1 \\ r, & \text { for } r+1 \leq x \leq k, \\ \operatorname{ex}_{\mathbb{Z}}(x, k, r), & \text { for } k \leq x\end{cases}
$$

Observe that $\operatorname{ex}_{\mathbb{Z}}(k, k, r)=r$, so that $h$ is well-defined. In the rest of this section we show that $h$ is a linear weighting function. Once we know this (via Lemmas 2, 3 and 4) the proof of Theorem 5 is immediate:

Proof of Theorem 5. Since $a-1 \leq(\ell+1)-2$ we have that $h(x, \ell+1, a-1)$ is a linear weighting function. Furthermore $h(\ell+1, \ell+1, a-1)=a-1$, so that we can apply Lemma 1 . Because $\lceil n / q\rceil \geq\lceil k / q\rceil=\ell+1$ we get that in the interesting range $h(x)=\operatorname{ex}_{\mathbb{Z}}(x, \ell+1, a-1)$ and the inequality follows.

In the rest of this section (and in the next section, too), we suppose that $r \leq k-2$. We obtain $p=0, q=1, a=r+1$ and $\ell=k-1$. First, we consider the case $2(r+1) \leq k$. 
Since this is the the case of Theorem 3, we have $h(n)=h_{r}(n)$ and there is nothing to prove.

Also for $2(r+1)>k$ and $n \leq 2(r+1)$ Theorem 4 can be applied and we get that $h(n)=h_{r+1, k-1}(n)$. Therefore all 3 properties (L1)-(L3) hold in this range.

Thus from now on we can assume that $n>2 r+2>k$ and $h(n)=\operatorname{ex}_{\mathbb{Z}}(n, k, r)$.

Notice that Theorem 2 yields (for all $0<r+1<k \leq n$ )

$$
\operatorname{ex}_{\mathbb{Z}}(n, k, r) \leq n+r-k<n-1 .
$$

Hence the Lipschitz property of $h$ is an immediate consequence of (12). We obtain

$$
\operatorname{ex}_{\mathbb{Z}}(n+1, k, r) \leq \operatorname{ex}_{\mathbb{Z}}(n, k, r)+\left\lfloor 2 \operatorname{ex}_{\mathbb{Z}}(n, k, r) /(n-1)\right\rfloor \leq \operatorname{ex}_{\mathbb{Z}}(n, k, r)+1 .
$$

For the other two properties (L1), (L3) we need to prove two Lemmas. For a given weighting $w$ let $h_{w}(x)=\max \{w(A):|A|=x\}$ and recall that the weight of a vertex was defined by $w(v)=w(\{u v: u \in V\})$.

Lemma 2. If $0<r+1<k<2 r+2, k \leq n, \operatorname{ex}_{\mathbb{Z}}(n, k, r)>\operatorname{ex}_{\mathbb{Z}}(n-1, k, r)$ and $w$ is a weighting of $K_{n}$ achieving $\operatorname{ex}_{\mathbb{Z}}(n, k, r)$ then every vertex has positive weight. Furthermore if $n>2 r+2$, then $0=h_{w}(1) \leq h_{w}(2) \leq \cdots \leq h_{w}(k)=r$.

Proof. For a given vertex $v$ the positivity of $w(v)$ follows from

$$
\operatorname{ex}_{\mathbb{Z}}(n, k, r)>\operatorname{ex}_{\mathbb{Z}}(n-1, k, r) \geq w\left(K_{n}-v\right)=\operatorname{ex}_{\mathbb{Z}}(n, k, r)-w(v) .
$$

So now assume that $n>2 r+2$. The first equality is trivial and the second one follows from the choice of $w$. For the chain of inequalities, suppose that $i$ is maximal such that $h_{w}(i)>h_{w}(i+1)$ and let $A$ be a set achieving $h_{w}(i)$. Then for every vertex $v \notin A$ we have $w(\{u v: u \in A\}) \leq h_{w}(i+1)-h_{w}(i)$, so that

$$
\begin{aligned}
i & \leq \sum_{u \in A} w(u) \leq 2 h_{w}(i)+(n-i)\left(h_{w}(i+1)-h_{w}(i)\right) \\
& =2 h_{w}(i+1)+(n-i-2)\left(h_{w}(i+1)-h_{w}(i)\right) \\
& \leq 2 r+(n-i-2)(-1)=i+(2 r+2-n)<i
\end{aligned}
$$

a contradiction.

Lemma 2 will enable us to prove

$$
\text { If } 2 r+2<n<m \text { and } h(n)>h(m) \text {, then also } h(n-1)>h(m) \text {. }
$$

But then the monotonicity of $h$ is straight forward. If $h(n+1)<h(n)$ then we can apply (16) repeatedly and obtain that $h(n+1)<h(2 r+2)$. This is a contradiction since we know that $h(n+1) \geq h_{r+1, k-1}(n+1) \geq h_{r+1, k-1}(2 r+2)=h(2 r+2)$. 
To prove (16) we may assume that $h(n-1)<h(n)$, since otherwise there is nothing to show. Let $w$ be any weighting achieving $h(n)$ and extend this to a weighting $w^{\prime}$ of $K_{m}$ by making all new edges weight 0 . Notice that for any set $A$ of $k$ vertices in $K_{m}$

$$
w^{\prime}(A)=w\left(A \cap K_{n}\right) \leq h_{w}\left(\left|A \cap K_{n}\right|\right) \leq r
$$

by Lemma 2 . Hence $w^{\prime}$ is $(k, r)$-dense contradicting $h(n)>h(m)$.

The rest of the section is devoted to the proof of property (L3). It is clear that in general we may not be able to take just any weighting achieving $\operatorname{ex}_{\mathbb{Z}}(n, k, r)$ for this purpose. For example we need $w(u v)=w(\{u, v\}) \leq h(2)=1$, so that we can't have multiple edges.

We call a maximal vertex set $A$ with the property $w(A) \geq|A|-1$ a component. Notice that components need not be disjoint, but every vertex is in some component since $w(\{v\})=0$.

Lemma 3. If $0<r+1<k<2 r+2<n$, $\operatorname{ex}_{\mathbb{Z}}(n, k, r)>\operatorname{ex}_{\mathbb{Z}}(n-1, k, r)$ and $w$ achieves $\operatorname{ex}_{\mathbb{Z}}(n, k, r)$ then every component $A$ has $|A| \leq r+1$ and $w(A)=|A|-1$.

Proof. Let $A$ be a component. Then (15) implies $|A|<k$ and by Lemma 2 we get that $|A| \leq r+1$. Next notice that for every vertex $v \notin A$

$$
w(\{u v: u \in A\})=w(A+v)-w(A) \leq(|A+v|-2)-w(A)=|A|-w(A)-1,
$$

so that for $w(A) \geq|A|$ we would obtain

$$
\begin{aligned}
w(A)+w(\{u v: u \in A, v \notin A\}) & \leq w(A)+(n-|A|)(|A|-w(A)-1) \\
& =(n-|A|)(|A|-1)-w(A)(n-|A|-1) \\
& \leq(n-|A|)(|A|-1)-|A|(n-|A|-1) \\
& =2|A|-n<0 .
\end{aligned}
$$

But that is impossible, since then $w\left(K_{n} \backslash A\right)>w\left(K_{n}\right)=\operatorname{ex}_{\mathbb{Z}}(n, k, r)$. Depending on $\left|K_{n} \backslash A\right|$ this would be a contradiction either to the monotonicity of $\operatorname{ex}_{\mathbb{Z}}(n, k, r)$ or Lemma 2.

Definition. A weighting $w$ is called simple if

(S1) $w(e) \leq 1$ for every edge $e$,

(S2) the edges of weight 1 induce a disjoint union of stars, and

(S3) $w(v)>0$, unless $v$ is isolated (i.e., only incident to edges of weight zero).

A set of of vertices $A$ is called simple if

(A1) $A$ induces a star with center $c$,

(A2) For $u \in A$ and $v \notin A$ we have $w(u v)=0$ unless $u=c$ and $w(c v)<0$, and

(A3) $w(c)>0$ for $|A|>1, w(c)=0$ otherwise. 
In a simple weighting the only edges of negative weight are between the centers of stars. For example the weightings provided for $h_{a}$ and $h_{a, \ell}$ are simple. Simple sets are always disjoint, so that a weighting is simple if and only if every vertex is in some simple set.

Lemma 4. If $0<r+1<k \leq n$, then $\operatorname{ex}_{\mathbb{Z}}(n, k, r)$ can be achieved by a simple weighting $w$. Moreover $h_{w}$ has the Lipschitz property and is non-decreasing.

Lemma 4 will enable us to verify the third property for $h$ by choosing $w$ to be the simple weighting guaranteed. Notice that $h$ was chosen to increase as much as possible initially. Therefore we get that $h_{w}(x) \leq h(x)$ for $x \leq k$, since $h_{w}$ also has the Lipschitz property, is non-decreasing and satisfies $h_{w}(k) \leq r=h(k)$. Thus for all sets $A$ of size at most $k$ we have $w(A) \leq h_{w}(|A|) \leq h(|A|)$. For $|A| \geq k$ we trivially get $w(A) \leq$ $\operatorname{ex}_{\mathbb{Z}}(|A|, k, r)=h(|A|)$.

Proof of Lemma 4. For a given simple weighting $w$ we see that $h_{w}(x+1) \leq h_{w}(x)+1$ by taking a maximum weight set of size $x+1$ and removing a leaf of one of the stars. If the set consisted only of centers then all internal edges were non-positive and we can remove any vertex. Similarly $h_{w}(x+1) \geq h_{w}(x)$ since we can take a set achieving $h_{w}(x)$ and add an isolated vertex or a leaf of some star. If only centers remain, then adding in any one of those will increase $h_{w}(x)$ by Property (S3). Thus it only remains to construct such a simple weighting $w$.

If $2 r+2 \leq k-1$ or $n \leq 2 r+2$ we can use the simple weightings from $h_{r+1}$ or $h_{r+1, k-1}$ respectively, so we may assume that $n>2 r+2>k$.

In the case when $\operatorname{ex}_{\mathbb{Z}}(n, k, r)=\operatorname{ex}_{\mathbb{Z}}(n-1, k, r)$ we use the simple weighting $w$ for $K_{n-1}$ and add in an isolated vertex to obtain the simple weighting $w^{\prime}$. We get $h_{w^{\prime}}(k)=$ $\max \left\{h_{w}(k), h_{w}(k-1)\right\}=h_{w}(k) \leq r$, so that $h_{w^{\prime}}$ achieves $\operatorname{ex}_{\mathbb{Z}}(n, k, r)$.

So we may also assume that $\operatorname{ex}_{\mathbb{Z}}(n, k, r)>\operatorname{ex}_{\mathbb{Z}}(n-1, k, r)$. Hence, by Lemma 2 , every vertex has positive weight and $h_{w}(x) \leq r$ for $x \leq k$ for every weighting achieving $\operatorname{ex}_{\mathbb{Z}}(n, k, r)$. Take a weighting $w$ achieving $\operatorname{ex}_{\mathbb{Z}}(n, k, r)$ with a minimum number of vertices not in any simple set. Let $v$ be such a vertex, since if there is no such vertex then $w$ is simple and we are done. Let $A$ be a component containing $v$, so by Lemma 3 $w(A)=|A|-1$. We will create a new weighting $w^{\prime}$ by making $A$ simple: Replace $w$ on $A$ by a star centered at $v$ and for $y \notin A$ defining $w^{\prime}(x y)=0$ if $x \in A-v$ and $w^{\prime}(v y)=\sum\{w(u y): u \in A\}=w(A+y)-w(A) \leq(|A+y|-2)-(|A|-1)=0$. Hence $w^{\prime}\left(K_{n}\right)=w\left(K_{n}\right)$ and $v$ is now in the simple set $A$. Furthermore if $S$ used to be a simple set, then $S \backslash A$ still is: if the center of $S$ was in $A$ then $S \subset A$, since $A$ was a component. Hence $S \backslash A$ is still a star. Furthermore all new edges from $S \backslash A$ are non-positive, and all leafs still have weight 0 on all outgoing edges.

Let $K$ be a set on $k$ vertices. If $v \notin K$, then

$$
w^{\prime}(K)=w^{\prime}(K \backslash A)=w(K \backslash A) \leq h_{w}(|K \backslash A|) \leq r .
$$


TURÁN PROBLEMS FOR INTEGER-WEIGHTED GRAPHS 23

If on the other hand $v \in K$, then let $K^{\prime}=K \cup A$ and observe that by (15)

$$
w^{\prime}(K)+\left|K^{\prime}\right|-k=w^{\prime}\left(K^{\prime}\right)=w\left(K^{\prime}\right) \leq\left|K^{\prime}\right|-k+r .
$$

In either case $w^{\prime}(K) \leq r$, so that $h_{w^{\prime}}(k) \leq r$, in contradiction to our choice of $w$.

\section{MORE ON THE LINEAR TERM}

The aim of this section is the proof of Theorem 6 . Theorem 5 suggests that to solve the problem of $\operatorname{ex}_{\mathbb{Z}}(n, k, r)$ for all values it will be crucial to study the purely linear term. Lemma 4 tells us what good constructions will essentially look like, and it also "simplifies" the proof of upper bounds. For example

Theorem 8. If $r \leq k-2$ then $\operatorname{ex}_{\mathbb{Z}}(n, k, r) \leq\left\lfloor\frac{r}{r+1} n\right\rfloor$ with equality when $r=k-2$.

Proof. Since $k \geq r+1$ the result is clear for $n=k$. For $n>k$ there is nothing to show when $\operatorname{ex}_{\mathbb{Z}}(n, k, r)=\operatorname{ex}_{\mathbb{Z}}(n-1, k, r)$. Otherwise we use Lemma 4 to obtain a simple weighting achieving $\operatorname{ex}_{\mathbb{Z}}(n, k, r)$ and observe that every simple set is also a component. Hence by Lemma 3 every one of the stars has at most $r+1$ vertices. Therefore the most edges we can have is taking disjoint stars on $r+1$ vertices without negative weights and this is reflected in the formula. Furthermore for $r=k-2$ this weighting is achievable.

We conjecture that the weighting function $g$ given by a disjoint union of one star on $v$ vertices and as many stars on $u$ vertices $(1 \leq u \leq v)$ as possible, plus one remainder star on at most $u$ vertices, is essentially optimal. (All other edges have weight 0 , i.e., this is a simple weighting as it was defined in the previous section). Let

$$
g(x, u, v):= \begin{cases}x-1, & \text { for } x \leq v \\ v-1+\left\lfloor\frac{u-1}{u}(x-v)\right\rfloor, & \text { for } v \leq x .\end{cases}
$$

Lemma 5. For the case $0<r+1<k<2 r+2$

$$
\operatorname{ex}_{\mathbb{Z}}(n, k, r) \geq \max \left\{h_{r+1, k-1}(n), g(n, u, v)\right\},
$$

where $u=\lfloor(k-1) /(k-r-1)\rfloor$ and $v=k-1-u(k-r-2)$.

Proof. It was shown in Section 8. with a construction that $h_{r+1, k-1}(n)$ is a lower bound. Concerning the second term, consider the simple weighting defined before the Lemma. Since $v-u=k-1-u(k-r-1) \geq 0$, we get $v \geq u$. Also we have $u \geq 1$. Finally, one can check that this is indeed $(k, r)$-dense by observing that for $x \geq v$

$$
g(x, u, v)=v-1+\left\lfloor\frac{u-1}{u}(x-v)\right\rfloor=r+\left\lfloor\frac{u-1}{u}(x-k+1)\right\rfloor,
$$




\section{JOURNAL OF GRAPH THEORY}

and that $k \geq v$, so that $g(k, u, v)=r$.

We believe that equality always holds. We were able to prove, with some case analysis, that for $k<11$ equality holds in Lemma 5 for all $n \geq k$. In the rest of this section we show that Lemma 5 also gives the optimum when $n$ is sufficiently large.

Proof of Theorem 6 . If $k \geq 2 r+2$, that is when $u=1$, then the result follows from Theorem 3. So we may assume that $0<r+1<k<2 r+2$ and hence $u \geq 2$.

By Lemma 4 there exists an optimal simple weighting $w$. As in Lemma 2 let $h(x)$ denote the maximum possible weight an $x$-element set can have, where we dropped the subscript, since the underlying weight function is now fixed. For example $h(0)=h(1)=$ $0, h(2)=1$. We already know from Lemma 5 that

$$
h(n)=\operatorname{ex}_{\mathbb{Z}}(n, k, r) \geq r+\left\lfloor\frac{u-1}{u}(n-k+1)\right\rfloor .
$$

The proof of the upper bound for $h(n)$ consists of two steps. First, we will prove in Lemma 6 that the weighting $w$ contains at least $k$ vertices in good $u$-stars, that is stars on $u$ vertices without negative edges at the center vertex. Then Lemma 7 below will finish the proof of Theorem 6 .

Lemma 6. If $0<r+1<k<2 r+2, n \geq 3 k r$ and $w$ is a simple weighting satisfying (17) then at least $k$ vertices are in good $u$-stars.

Proof. Let $\mathcal{A}$ be the set of components (in the sense of Lemma 3) given by $w$. Every component $A \in \mathcal{A}$ induces a star and we denote its center by $v_{A}$. Furthermore, let $n_{A}$ denote the absolute value of the total sum of negative weights of the edges adjacent to the center $v_{A}$. The total contribution of the edges with at least one vertex in $A$ to the weight of $K_{n}$ is exactly $w\left(v_{A}\right)=(|A|-1)-n_{A}$. For components on more than one vertex this should be strictly positive, hence

$$
n_{A} \leq|A|-2 \text { for }|A| \geq 2 .
$$

From Lemma 4 we know that the function $h(x)$ is non-decreasing with $h(k) \leq r$, so that every star has at most $r$ edges, that is $|A| \leq r+1$. Now divide $\mathcal{A}$ into four parts:

$\mathcal{A}_{0}=\{A \in \mathcal{A}:|A|<u\}$ (small stars, including isolated vertices),

$\mathcal{A}_{1}=\left\{A \in \mathcal{A}:|A|=u\right.$ and $\left.n_{A}>0\right\}$ (bad $u$-stars),

$\mathcal{A}_{2}=\left\{A \in \mathcal{A}:|A|=u\right.$ and $\left.n_{A}=0\right\}$ (good $u$-stars), and

$\mathcal{A}_{3}=\{A \in \mathcal{A}:|A|>u\}$ (big stars).

Let $V_{i}=\cup \mathcal{A}_{i}$ be the set of all the vertices in the corresponding part. Our first aim is to prove that there are only few big stars:

$$
\left|V_{3}\right| \leq r(k-1) .
$$




\section{TURÁN PROBLEMS FOR INTEGER-WEIGHTED GRAPHS 25}

Indeed, split $\mathcal{A}$ into $r$ parts $\mathcal{B}_{1}, \ldots, \mathcal{B}_{r}$ such that each $\mathcal{B}_{i}$ contains no negative edge. This can be done, since by (18) $n_{A} \leq r-1$. (We can define these parts recursively, putting the first member of $\mathcal{A}$ into $\mathcal{B}_{1}$. So suppose we have already split some members of $\mathcal{A}$. For the next member, call it $A$, we can always find some $\mathcal{B}_{i}$ such that $A$ is not joined to $\cup \mathcal{B}_{i}$ by a negative edge, since at most $r-1$ negative edges are incident to $v_{A}$ ). We claim that the members of $\mathcal{B}_{i} \cap \mathcal{A}_{3}$ cover at most $k-1$ points, which immediately gives (19).

Indeed, suppose to the contrary that there exist $A_{1}, \ldots, A_{t} \in \mathcal{B}_{i} \cap \mathcal{A}_{3}$ such that $\left|A_{1} \cup \cdots \cup A_{t}\right| \geq k$. Consider a minimal such union, i.e., $\left|A_{1} \cup \cdots \cup A_{t-1}\right|<k$. Then there exists a $k$-set $K$ containing $A_{1} \cup \cdots \cup A_{t-1}$ and the center of $A_{t}$ and contained in $A_{1} \cup \cdots \cup A_{t}$. Thus $w(K)=k-t$. This is at most $r$, implying that $t-1 \geq k-r-1$ and hence

$$
\left|A_{1} \cup \cdots \cup A_{t-1}\right| /(t-1) \leq(k-1) /(k-r-1) .
$$

This implies that $\left|A_{i}\right| \leq u$ (for some $i \leq t-1$ ), a contradiction.

Next we observe that for all stars $A \notin \mathcal{A}_{3}$ we have $w(A) \leq(u-1) A / u$. Therefore we get that $w\left(B_{i}\right) \leq g\left(\left|B_{i}\right|, u, v\right)$, where $B_{i}$ stands for the vertex set $\cup \mathcal{B}_{i}$. Taking into account the small members of $\mathcal{B}_{i}$, we actually obtain

$$
\begin{aligned}
w\left(B_{i}\right) & \leq g\left(\left|B_{i} \backslash V_{0}\right|, u, v\right)+\frac{u-2}{u-1}\left|B_{i} \cap V_{0}\right| \\
& \leq \frac{u-1}{u}\left|B_{i}\right|+(k-r-1) / u-\left|B_{i} \cap V_{0}\right| / u(u-1),
\end{aligned}
$$

since $r-\frac{u-1}{u}(k-1) \leq(k-r-1) / u$.

Adding up these inequalities for all $1 \leq i \leq r$ and considering the fact that every member in $\mathcal{A}_{1}$ is adjacent to a negative edge we get

$$
h(n)=w\left(K_{n}\right) \leq \frac{u-1}{u} n+r(k-r-1) / u-\left|V_{0}\right| \frac{1}{u(u-1)}-\frac{1}{2}\left|V_{1}\right| / u .
$$

This upper bound and the lower bound (17) yields $\left|V_{0} \cup V_{1}\right| \leq 2 k r-k+r$. Together with (19) we obtain that $\left|V_{2}\right| \geq n-3 k r+k \geq k$, the desired $k$ vertices in good $u$-stars.

Lemma 7. If $0<r+1<k<2 r+2, k \leq n$ and $w$ is an optimal simple weighting containing at least $k$ vertices in good $u$-stars, then for all $0 \leq x \leq n$

$$
h_{w}(x) \leq r+\left\lfloor\frac{u-1}{u}(x-k+1)\right\rfloor .
$$

Proof. We proceed by induction on $x$. For $x=0$ a simple computation yields that $0 \leq r+\lfloor(u-1)(-k+1) / u\rfloor$. For $x>0$ it is sufficient to prove the upper bound for numbers of the form $x=k+j u$ where $j$ is a (not necessarily positive) integer, i.e.,

$$
h(k+j u) \leq r+j(u-1) .
$$


Indeed, the Lipschitz property of $h$ (implied by Lemma 4), implies the remaining values.

So consider a set $K$ on $k+j u$ elements with $w(K)=h(k+j u)$. We may suppose that $K$ meets the minimum number of stars, say with vertex sets $A_{1}, \ldots, A_{c}$. Abbreviate the center of $A_{i}$ by $v_{i}$ and observe that each $v_{i}$ is contained in $K$ (for $1 \leq i \leq c$ ). Indeed, if $v_{i} \notin K$, but $y \in A_{i} \cap K$ then $w(K-y)=w(K)$, and the result follows by induction. Thus we can also assume that $A_{1} \cup \cdots \cup A_{c-1} \subset K \subset A_{1} \cup \cdots \cup A_{c}$.

First we prove (20) for $j \leq 0$. Observe that by the choice of $K$ and the fact that there are at least $k$ vertices in good $u$-stars we can find $|j| \operatorname{good} u$-stars $A_{1}^{\prime}, \ldots A_{|j|}^{\prime}$ disjoint from $K$. As $w(K)+|j|(u-1)=w\left(K \cup A_{1}^{\prime} \cup \cdots \cup A_{|j|}^{\prime}\right) \leq r$ we obtain (20).

To prove (20) for positive $j$, suppose to the contrary, that $w(K)>r+j(u-1)$. By hypothesis we have $h(k+(j-1) u) \leq r+(j-1)(u-1)$. Then the Lipschitz property implies that $h(k+j u) \leq h(k+(j-1) u)+u$, hence we have $w(K)=r+j(u-1)+1$ and $h(k+(j-1) u+i)=r+(j-1)(u-1)+i$ for $0 \leq i \leq u$.

Let $M=A_{1} \cup \cdots \cup A_{c}$. This set contains $K$, and we let $|M|=|K|+m$. As $M$ can be obtained from $K$ by adding $m$ vertices of degree (and weight) one we have that $w(M)=w(K)+m$. Then, again, the Lipschitz property implies that

$$
h(k+(j-1) u+i)=r+(j-1)(u-1)+i \quad \text { for all } \quad 0 \leq i \leq u+m .
$$

Let $q_{i}$ denote the sum of the absolute values of the weights of the edges from $v_{i}$ to the other centers $v_{t}(1 \leq t \leq c)$. We have that $w\left(M \backslash A_{i}\right)=w(M)-\left(\left|A_{i}\right|-1\right)+q_{i}>$ $w(M)-\left|A_{i}\right|$. This and (21) imply that $\left|A_{i}\right|>u+m$. Thus we can use the hypothesis that our result is true for $M \backslash A_{i}$. We obtain that

$$
\begin{aligned}
r+j(u-1)+m+1 & =h(|M|)=w(M)=w\left(M \backslash A_{i}\right)+\left(\left|A_{i}\right|-1\right)-q_{i} \\
& \leq r+\left\lfloor\frac{u-1}{u}\left(k+j u+m-\left|A_{i}\right|-(k-1)\right)\right\rfloor+\left|A_{i}\right|-1-q_{i} .
\end{aligned}
$$

Multiplying by $u$, dropping the floors, and rearranging terms we get that

$$
u+1+m+u q_{i} \leq\left|A_{i}\right| .
$$

Adding this up for all $i$ we obtain

$$
c(u+1)+c m+2 u Q \leq \sum\left|A_{i}\right|=k+j u+m,
$$

where $Q$ is the absolute value of $w\left(\left\{v_{1}, \ldots, v_{c}\right\}\right)$. On the other hand, from (21) and from the structure of $M$ we can calculate $Q$ as follows:

$$
r+j(u-1)+1+m=w(M)=|M|-c-Q=k+j u+m-c-Q .
$$


TURÁN PROBLEMS FOR INTEGER-WEIGHTED GRAPHS 27

Rearranging we have $Q+c=(k-r-1)+j$. Subtracting $(u+1)$ times this equality from the two sides of $(22)$ we obtain that

$$
(c-1) m+Q(u-1)+(j-1) \leq(k-1)-(u+1)(k-r-1) .
$$

However, here on the left-hand side all the three terms are non-negative $(c, u, j \geq 1$ and $m, Q \geq 0)$ and the right hand side is negative. This contradiction shows that our assumption $h(k+j u)>r+j(u-1)$ was wrong, finishing the proof of $(20)$.

\section{DENSITY PROBLEMS FOR MULTIGRAPHS}

In this section we return to our starting point. We will determine as a corollary the asymptotic density for non-negative integer weights for infinitely many cases. We investigate $\operatorname{ex}_{W}(n, k, r)$ for $W=\mathbb{N}$ and for $W=[i]:=\{0,1,2, \ldots, i\}$. Let $\|M(p / q)\|$ denote the absolute value of the smallest (non-positive) entry of the matrix $M(p / q)$ defined in Section 7 .

Theorem 9. Suppose that $r=r^{\prime}+j\left(\begin{array}{c}k \\ 2\end{array}\right)$ with $0 \leq r^{\prime}<\left(\begin{array}{c}k \\ 2\end{array}\right), m\left(k, r^{\prime}\right)=p / q$ and $\|M(p / q)\| \leq j$. Then

$$
\operatorname{ex}_{\mathbb{N}}(k, r)=\operatorname{ex}_{[j+1]}(k, r)=j+p / q .
$$

Proof. Indeed, the upper bound of Theorem 2 is also valid for these cases. The lower bound follows from the observation that the construction in Lemma 1 (using the zero function for $h$ ) has maximum weight 1 and minimum weight $-\|M(p / q)\|$ and we can add weight $j$ to every edge.

In fact, we have a stronger result. In the range of Theorem 9 we have

lower bound of Lemma $1 \leq \operatorname{ex}_{[j+1]}(n, k, r) \leq \operatorname{ex}_{\mathbb{N}}(n, k, r) \leq$ upper bound of Theorem 2 .

Here the gap between the upper and lower bounds is less than $n$.

It can be checked that for $0 \leq p<q$

$$
\|M(p / q)\| \leq q-p-1 .
$$

This is best possible for example when $p=1$ or $p=q-1$, but in general we expect that the negative weight is only $\mathrm{O}(\log q)$.

Proof of (24). Induction on $p$. For $p=0$ and $q=1$ the construction has all weights 0 and the bound holds with equality. For $p>0$, let $q=(t+2) p-p^{\prime}$ with $0 \leq p^{\prime}<p$. We have a negative weight of at most $\left\|M\left(p^{\prime} / p\right)\right\| \leq p-p^{\prime}-1$. Thus, for $0<p<q$, we have a negative weight of at most $p-p^{\prime}-1+t \leq p(t+2)-p^{\prime}-p-1=q-p-1$. 
A $(k, r)$-dense graph $G$ for $W=\mathbb{N}$ has no edges of weight more than $r$. The following observation says that its maximum weight can be bounded by $\ell:=\left\lfloor r /\left\lfloor k^{2} / 4\right\rfloor\right\rfloor$.

$$
\operatorname{ex}_{\mathbb{N}}(k, r)=\operatorname{ex}_{[\ell]}(k, r)
$$

Proof. Indeed, let $H$ be the graph formed by the edges of weight at least $\ell+1$. This graph does not contain the complete bipartite graph $K_{\lfloor k / 2\rfloor,\lceil k / 2\rceil}$ as a subgraph. Hence a theorem of Kővári, T. Sós and Turán [32] implies that $|E(H)|=\mathrm{O}\left(n^{1-2 / k}\right)$. So the edges of high weight contribute only o $\left(n^{2}\right)$ to the total weight of $G$.

One can formulate the following consequence of (25).

$$
\operatorname{ex}_{\mathbb{N}}(k, r)=\operatorname{ex}_{[2 i-1]}(k, r) \quad \text { for } \quad r \leq i\left(\begin{array}{l}
k \\
2
\end{array}\right) .
$$

We conjecture that $2 i-1$ can be replaced by $i$ or $i+1$.

For fixed $k$ Theorem 9 immediately implies the value of $\operatorname{ex}_{\mathbb{N}}(k, r)$ for all but finitely many $r$. For values $k-2<r<\left\lfloor k^{2} / 4\right\rfloor(25)$ implies that $\operatorname{ex}_{\mathbb{N}}(k, r)=0$, while $\operatorname{ex}_{\mathbb{Z}}(k, r) \geq$ $1 /(k-1)$. So for these values of $(k, r)$ negative weights help. The first such case is $(k, r)=(4,3)$, given by $M(1 / 3)$.

We close this Section by reviewing $\operatorname{ex}_{\mathbb{N}}(n, k, r)$ for $k \leq 5$.

The cases for $k=3$ have been settled in [5] where it is shown that $\operatorname{ex}(n, 3,1)=\lfloor n / 2\rfloor$ and $\operatorname{ex}(n, 3,2)=\left\lfloor n^{2} / 4\right\rfloor$ which together with the trivial $\operatorname{ex}(n, 3,0)=0$ shows that all these values can be obtained by non-negative weights: A matching, $T_{n, 2}$, the empty graph and for higher values of $r$ we take translates of those.

For $k=4$ and $r \leq 3$ we can have at most one edge of weight 2 in the positive weight case. $\operatorname{ex}_{\mathbb{N}}(n, 4,0)=0, \operatorname{ex}_{\mathbb{N}}(n, 4,1)=1$ and $\operatorname{ex}_{\mathbb{N}}(n, 4,2)=\lfloor 2 n / 3\rfloor$ follows immediately. For $r=3$ we also get that the weight 1 edges cannot contain a $C_{4}$, so that $\operatorname{ex}_{\mathbb{N}}(n, 4,3) \leq$ $\operatorname{ex}\left(n, C_{4}\right)=\frac{1}{2} n^{3 / 2}+\mathrm{O}\left(n^{4 / 3}\right)$ by a result of Erdős, Rényi and Sós [21] and independently Brown [6]. Hence $\operatorname{ex}_{\mathbb{N}}(4, r)=0$ for all $r \leq 3$, but $\operatorname{ex}_{\mathbb{Z}}(4,3)=1 / 3$ which is the only case for $k=4$ with $\operatorname{ex}_{\mathbb{N}}(k, r)<\operatorname{ex}_{Z}(k, r)$. For all the remaining cases $r>4$ we get equality here, e.g., $\operatorname{ex}_{\mathbb{Z}}(4,4)=1 / 2=\operatorname{ex}_{\mathbb{N}}(4,4)$ and $\operatorname{ex}_{\mathbb{Z}}(4,5)=2 / 3=\operatorname{ex}_{\mathbb{N}}(4,5)$.

For $k=5$, the only cases where $\operatorname{ex}_{\mathbb{Z}}(5, r)$ could be bigger than $\operatorname{ex}_{\mathbb{N}}(5, r)$ are $r=$ $4,5,14$. The arguments for $r=4,5$ are similar to those above as the edges of weight 1 cannot contain a $K_{2,3}$, so that $\operatorname{ex}_{\mathbb{N}}(5,4)=\operatorname{ex}_{N}(5,5)=0$, whereas $\operatorname{ex}_{\mathbb{Z}}(5,4)=1 / 4$ and $\operatorname{ex}_{\mathbb{Z}}(5,5)=1 / 3$. Finally, we have $\operatorname{ex}_{\mathbb{Z}}(5,14)=5 / 4$ while one can prove that $\operatorname{ex}_{\mathbb{N}}(5,14)=$ $\operatorname{ex}_{\{0,1,2\}}(5,14)=7 / 6$. But this, and other results about $\operatorname{ex}_{\mathbb{N}}$, are the subject of another paper [25].

\section{OPEN QUESTIONS}

Besides the obvious open questions concerning $\operatorname{ex}_{Z}(n, k, r)$ (e.g., closing the $\mathrm{O}(n)$ gap, determining the exact values for small $n$ ) one can ask what we can say about extremal 
graphs. The results from [5] show that even in the simplest nontrivial case (i.e. $k=3$ ) there can be many different extremal graphs. In our proof of the general result we use induction, and it looks very difficult to build the extremal structure into the induction hypothesis. So to study the exact structure of the extremal graphs new ideas will be needed.

\section{ACKNOWLEDGMENTS}

The research of the first author was supported in part by the Hungarian National Science Foundation grant OTKA 016389, and by a National Security Agency grant MDA90498-I-0022. The research of the second author was done in part as a research assistant at the University of Illinois and in part while visiting Universität Ulm. The authors are indebted to M. Simonovits and R. Ramamurthi for helpful comments.

\section{References}

[1] R. Ahlswede, N. Cai, Z. Zhang, Rich colorings with local constraints, J. Combin. Inform. System Sci. 17 (1992), 203-216.

[2] M. Axenovich, A. Kündgen, On a generalized anti-Ramsey problem, Combinatorica 21 (2001), 335-349.

[3] B. Bollobás, Extremal Graph Theory, Academic Press, New York/London (1978).

[4] A. Bondy and M. Simonovits, Cycles of even length in graphs, Journal of Combinatorial Theory, Ser. B 16 (1974), 97-105.

[5] J. A. Bondy, Zs. Tuza, A weighted generalization of Turán's theorem, J. Graph Theory 25 (1997), 267-275.

[6] W. G. Brown, On graphs that do not contain a Thomsen graph, Canad. Math. Bull. 9 (1966), 281-285.

[7] W. G. Brown, P. Erdős, M. Simonovits, Extremal problems for directed graphs, J. Combinatorial Theory, Ser. B 15 (1973), 77-93.

[8] W. G. Brown, P. Erdős, M. Simonovits, Multigraph extremal problems, Colloques Internationaux CNRS 260 (1978), 63-66.

[9] W. G. Brown, P. Erdős, M. Simonovits, Inverse extremal digraph problems, Colloq. Math. Soc. János Bolyai 37, North-Holland, Amsterdam - New York (1984), 119-156.

[10] W. G. Brown, P. Erdős, M. Simonovits, Algorithmic solution of extremal digraph problems, Trans. Amer. Math. Soc. 292 (1985), 421-449.

[11] W. G. Brown, P. Erdős, V. T. Sós, Some extremal problems on r-graphs, New directions in the theory of graphs (Proc. Third Ann Arbor Conf., Univ. Michigan, Ann Arbor, Mich, 1971), 53-63, Academic Press, New York (1973). 
[12] W. G. Brown, P. Erdős, V. T. Sós, On the existence of triangulated spheres in 3-graphs, and related problems, Period. Math. Hungar. 3 (1973), 221-228.

[13] W. G. Brown, M. Simonovits, Digraph extremal problems, hypergraph extremal problems, and the densities of graph structures, Discrete Math. 48 (1984), 147-162.

[14] D. de Caen, Z. Füredi, The maximum size of 3-uniform hypergraphs not containing a Fano plane, J. Combin. Theory Ser. B 78 (2000), 274-276.

[15] D. de Caen, L. Székely, On a problem of Erdős and Lovász, Ars Combin. 35 (1993), 187-192.

[16] G. Dirac, Extensions of Turán's theorem on graphs, Acta Math. Acad. Sci. Hungar. 14 (1963), 417-422.

[17] P. Erdős, Extremal problems in graph theory, Theory of Graphs and its Applications (Proc. Sympos. Smolenice, 1963), 29-36, Publ. House Czechoslovak Acad. Sci., Prague (1964).

[18] P. Erdős, Extremal problems in graph theory, A Seminar on Graph Theory, 54-59, Holt, Rinehart and Winston, New York ((1967) 1971).

[19] P. Erdős, R. Faudree, A. Jagota, T. Łuczak, Large subgraphs of minimal density or degree, J. Combin. Math. Combin. Comput. 22 (1996), 87-96.

[20] P. Erdös, P. Frankl, V. Rödl, The asymptotic number of graphs not containing a fixed subgraph and a problem for hypergraphs having no exponent, Graphs and Combinatorics 2 (1986), 113-121.

[21] P. Erdős, A. Rényi, V. T. Sós, On a problem of graph theory, Studia Sci. Math. Hungar. 1 (1966), 215-235.

[22] P. Erdős, M. Simonovits, A limit theorem in graph theory, Studia Sci. Math. Hungar. 1 (1966), 51-57.

[23] P. Erdős, A. H. Stone, On the structure of linear graphs, Bull. Amer. Math. Soc. 52 (1946), 1087-1091.

[24] Z. Füredi, M. X. Goemans, D. J. Kleitman, On the maximum number of triangles in wheel-free graphs, Combin. Probab. Comput. 3 (1994), 63-75.

[25] Z. Füredi, M. Simonovits, in preparation.

[26] Ira M. Gessel, Robert Indik, A recurrence associated with extremal problems, preprint, 9 pages (1989).

[27] A. I. Gol'berg, V. A. Gurvich, On the maximum number of edges for a graph with $n$ vertices in which every subgraph with $k$ vertices has at most $l$ edges, Soviet Math. Dokl. 35 (1987), 255-260.

[28] J. R. Griggs, M. Simonovits, G. R. Thomas, Extremal graphs with bounded densities of small subgraphs, J. Graph Theory 29 (1998), 185-207.

[29] V. A. Gurvich, Extremal homogeneous hypergraphs and a hypercombinatorial function, Soviet Math. Dokl. 37 (1988), 146-151.

[30] R. K. Guy, Sequences associated with a problem of Turán and other problems, Combinatorial theory and its applications, II. (Proc. Colloq., Balatonfüred, 1969), North-Holland, Amsterdam (1970), 553-569. 


\section{TURÁN PROBLEMS FOR INTEGER-WEIGHTED GRAPHS 31}

[31] W. Imrich, Explicit construction of graphs without small cycles, Combinatorica 4 (1984), 53-59.

[32] T. Kővári, V. T. Sós, P. Turán, On a problem of K. Zarankiewicz, Colloquium Math. 3 (1954), 50-57.

[33] J. Kuchenbrod, Extremal problems on weighted graphs, Ph.D. thesis, University of Kentucky (1999).

[34] F. Lazebnik, V. A. Ustimenko and A. J. Woldar, A new series of dense graphs of high girth, Bull. Amer. Math. Soc. 32 (1995), 73-79.

[35] A. Lubotzky, R. Phillips and P. Sarnak, Ramanujan graphs, Combinatorica 8 (1988), 261277.

[36] W. Mantel, Problem 28, Wiskundige Opgaven 10 (1907), 60-61.

[37] G. A. Margulis, Explicit construction of graphs without short cycles and low density codes, Combinatorica 2 (1982), 71-78.

[38] T. S. Motzkin, E. G. Straus, Maxima for graphs and a new proof of a theorem of Turán, Canad. J. Math. 17 (1965), 533-540.

[39] V. Rödl, A. Sidorenko, On the jumping constant conjecture for multigraphs, J. Combinatorial Theory, Ser. A 69 (1995), 347-357.

[40] I. Ruzsa, E. Szemerédi, Triple systems with no six points carrying three triangles, Colloq. Math. Soc. János Bolyai 18, North-Holland, Amsterdam - New York (1978), 939-945.

[41] B. S. Stechkin, On a surprising fact in extremal set theory, J. Combin. Theory Ser. A 29 (1980), 368-369.

[42] B. S. Stechkin, P. Frankl, Local Turán property for k-graphs, Russian, Mat. Zametki 29 (1981), 83-94.

[43] P. Turán, On an extremal problem in graph theory, Math. Fiz. Lapok, Hungarian 48 (1941), 436-452.

[44] P. Turán, On the theory of graphs, Colloquium Math. 3 (1954), 19-30.

[45] R. Wenger, Extremal graphs with no $C^{4}$ 's, $C^{6}$ 's or $C^{10}$ 's, Journal of Combinatorial Theory, Ser. B 52 (1991), 113-116. 